\title{
Effects of long-term and brain-wide colonization of peripheral bone marrow- derived myeloid cells in the CNS
}

\author{
Lindsay A. Hohsfield', Allison R. Najafi', Yasamine Ghorbanian², ${ }^{1,3}$ Neelakshi Soni ${ }^{1}$, Edna E. Hingco', Sung Jin Kim',
} Ayer Darling Jue', Vivek Swarup ${ }^{1}$, Mathew A. Inlay ${ }^{2,3}$ and Kim N. Green ${ }^{1 *}$ (D)

\begin{abstract}
Background: Microglia, the primary resident myeloid cells of the brain, play critical roles in immune defense by maintaining tissue homeostasis and responding to injury or disease. However, microglial activation and dysfunction has been implicated in a number of central nervous system (CNS) disorders, thus developing tools to manipulate and replace these myeloid cells in the CNS is of therapeutic interest.

Methods: Using whole body irradiation, bone marrow transplant, and colony-stimulating factor 1 receptor inhibition, we achieve long-term and brain-wide ( $~ 80 \%)$ engraftment and colonization of peripheral bone marrow-derived myeloid cells (i.e., monocytes) in the brain parenchyma and evaluated the long-term effects of their colonization in the CNS.

Results: Here, we identify a monocyte signature that includes an upregulation in Ccr1, Ms4a6b, Ms4a6c, Ms4a7, Apobec1, Lyz2, Mrc1, Tmem221, Trr8, Lilrb4a, Msr1, Nnt, and Wdfy1 and a downregulation of Siglech, S/c2a5, and Cc121a/b. We demonstrate that irradiation and long-term ( 6 months) engraftment of the CNS by monocytes induces brain regiondependent alterations in transcription profiles, astrocytes, neuronal structures, including synaptic components, and cognition. Although our results show that microglial replacement with peripherally derived myeloid cells is feasible and that irradiationinduced changes can be reversed by the replacement of microglia with monocytes in the hippocampus, we also observe that brain-wide engraftment of peripheral myeloid cells (relying on irradiation) can result in cognitive and synaptic deficits.

Conclusions: These findings provide insight into better understanding the role and complexity of myeloid cells in the brain, including their regulation of other CNS cells and functional outcomes.
\end{abstract}

Keywords: Microglia, Monocytes, CSF1R inhibition, Irradiation, Bone marrow transplant, Brain

\section{Background}

Microglia are the primary resident immune cells of the brain and possess the ability to rapidly proliferate and migrate in response to damage, injury, or infection. These immune detection and defense functions place microglia at the frontline of repair and recovery in the central nervous system (CNS). Microglia constitute $5-10 \%$ of all

\footnotetext{
* Correspondence: kngreen@uci.edu

'Department of Neurobiology and Behavior, University of California, 3208 Biological Sciences III, Irvine, CA 92697-4545, USA

Full list of author information is available at the end of the article
}

brain cells and are spatially arranged in a tile-like manner throughout the parenchyma-each cell inhabits a discrete territory in which its processes constantly retract and extend to survey the local environment [1], detecting and responding to stimuli accordingly. Microglia derive from yolk sac primitive macrophages, which enter and colonize the brain as immature microglia during embryonic development ( E9.5) [2, 3]. At E13.5, formation of the bloodbrain barrier (BBB) effectively isolates the brain, and thus from this point on and throughout adulthood, microglial population maintenance relies on local self-proliferation 
(under steady state conditions), independent of contributions from bone marrow (BM)-derived myeloid cells. Under inflammatory or disease conditions, studies have shown that BM-derived peripheral myeloid cells (i.e., monocytes) enter the brain, but do not differentiate into microglia or contribute significantly to the resident CNS microglial pool unless under exceptional circumstances (i.e., BM transplantation) [2, 4, 5]. However, even under these circumstances (i.e. BM transplant models), only a fraction of microglia derive from BM sources [6].

Microglia are dependent on signaling through the colony-stimulating factor 1 receptor (CSF1R) for their survival across the lifespan. We previously identified several selective CSF1R inhibitors that are orally bioavailable and CNS-penetrant and demonstrated that administration of these inhibitors results in rapid elimination of the murine microglial compartment [7-10]. Investigations on the effects of CSF1R inhibitors in primates [11] and humans [12,13] are ongoing, but provide a promising therapeutic strategy for brain disorders affected by microglial dysfunction [14]. One of the inhibitors that we first identified, pexidartinib (PLX3397), recently received FDA approval for the treatment of tenosynovial giant cell tumor [15].

Due to their central role in the CNS and several brain disorders, microglia have become attractive therapeutic targets; thus, strategies that replace or manipulate these cells could provide broad-reaching implications. In addition to depletion, we previously demonstrated that following microglial elimination, withdrawal of CSF1R inhibitors stimulates the rapid repopulation of the entire CNS with new microglia, and that these new cells derive from surviving cells within the CNS [7, 16-21]. This remarkable ability to deplete and then rebuild a cellular compartment throughout the brain parenchyma could provide a unique tool for developing cell and gene-based delivery systems to the brain. However, in previously utilized chimeric paradigms, we have found that transplanted exogenous myeloid cells are rapidly outcompeted by the surviving endogenous microglia. Engraftment of myeloid cells is severely limited in the brain unless performed under conditions inducing a depleted microglial niche (i.e., genetic loss of CSF1R), limiting the effectiveness and scope of transplanted engineered cells [22-25].

BM transplantations are often clinically utilized to replace the BM compartment with healthy donor BM cells. This technique typically relies on whole body irradiation to achieve myeloablation; however, recent studies have also indicated that myelosuppressive agents, such as the chemotherapeutic busulfan, offer viable alternatives [26]. We and others recently learned that combining CSF1Rdependent microglial elimination with whole body irradiation stimulates the complete repopulation of the CNS myeloid niche with BM-derived cells [22]. This technique not only provides us with a novel and clinically relevant tool to fully replace the microglial tissue with exogenous myeloid cells, but also allows for the potential unprecedented delivery of engineered myeloid cells to the brain (e.g., to carry a corrected mutation or therapeutic payload). Here, we sought to evaluate and characterize this method of complete microglial cell replacement utilizing CSF1R inhibition and BM transplantation and the longterm effects peripheral myeloid cells confer in the brain.

The role of peripheral-derived monocytes in diseaseassociated pathologies and neurological disorders remains under debate $[27,28]$. Several studies have shown that monocyte infiltration into the brain is associated with injury, disease, and neuropsychiatric conditions, suggesting that monocytes contribute to neuronal injury and demyelination [29-34]. However, other studies have shown that peripheral myeloid cell engraftment after BM transplant is beneficial and in some diseases macrophages provide neuroprotection and promote recovery [35-40].

To understand how peripherally derived CNS-resident myeloid cells differ from resident microglia in terms of their effects on the brain, and as a proof-of-principle for a clinically feasible cell delivery system, we utilized a unique experimental paradigm combining whole body irradiation, BM transplant, and a CSF1R inhibitor (CSF1Ri) to investigate (1) whether full replacement of the microglial tissue can be achieved with BM-derived peripheral myeloid cells and (2) what are the long-term ( $\sim 10$ months after transplant; $\sim 6$ months after CSF1Ri treatment) consequences of this replacement on other CNS cell properties and structures, gene expression, and behavior and cognition.

\section{Methods}

\section{Compounds}

Pexidartinib (PLX3397) was provided by Plexxikon Inc. and formulated in AIN-76A standard chow at a dose of 600 ppm by Research Diets Inc.

\section{Mice}

All mice were obtained from The Jackson Laboratory. For transplant studies, bone marrow cells were isolated from CAG-EGFP mice (006567). All other mice were male C57BL/6 (000664) mice. Animals were housed with open access to food and water under $12 \mathrm{~h} / 12 \mathrm{~h}$ lightdark cycles. All mice were aged to 1.5 months unless otherwise indicated.

\section{Animal treatments}

All rodent experiments were performed in accordance with animal protocols approved by the Institutional Animal Care and Use Committee at the University of California, Irvine (UCI). Microglial depletion: Mice were administered ad 
libitum with PLX3397 at a dosage of 600 ppm (to eliminate microglia) or vehicle (control) for 14 days. Bone marrow transplant: C57BL/6 mice were anesthetized with isoflurane and then irradiated with $1000 \mathrm{cGy}$ (whole-body irradiated) and reconstituted via retroorbital injection with $2 \times 10^{6}$ whole BM cells from CAG-EGFP mice. Blood was measured at $\sim 12$ weeks post-transplantation to track granulocyte chimerism. At the time of sacrifice, the mice were euthanized and BM was harvested and analyzed by flow cytometry for HSC chimerism. This established an average percent chimerism of $97 \%$ in all whole-body irradiated mice. Tissue collection: Following any treatments, mice were sacrificed via carbon dioxide inhalation and perfused transcardially with $1 \mathrm{X}$ PBS. The brains were extracted and dissected down the midline, with one half flash-frozen for subsequent RNA and protein analyses and the other half drop-fixed in $4 \%$ paraformaldehyde. Fixed brains were cryopreserved in PBS $+0.05 \%$ sodium azide $+30 \%$ sucrose, frozen, and sectioned at $40 \mu \mathrm{m}$ on a Leica SM2000 R sliding microtome for subsequent immunohistochemical analyses.

\section{Flow cytometry}

Bone marrow/hematopoietic stem cells were extracted from femurs and tibia by flushing with PBS $+2 \%$ FBS. Peripheral blood cells/granulocytes were collected via the tail vein in EDTA. Red blood cells were lysed using 1x ACK Lysis Buffer. Cells were then stained for analysis by flow cytometry with the following surface antibodies purchased from Biolegend at 1:200 unless otherwise indicated: CD34-eFlour660 (1:50, 50-0341-80, eBioscience), Sca-1AF700 (1:100, 108141), Ter119-PE/Cy5 (116209), ckit/ CD117-PE/Cy7 (25-1171-81, eBioscience), CD150/SLAMPerCP-eFlour710 (46-1502-82, eBioscience), CD11b-APC (101212), Gr1-AF700 (108422), CD45-APC/Cy7 (103116), NK1.1-PE (108707), and CD27-APC/Cy7 (124226). Flow cytometry analysis was performed using the BD LSRII.

\section{Behavioral and cognitive testing}

Mouse behavior, motor function, and cognition was evaluated using the following tasks: elevated plus maze, open field, novel place recognition test, rotarod, sociability test, spontaneous alternation Y-maze, and contextual fear conditioning in the order listed, and as previously described unless otherwise indicated [7, 8, 16]. Testing was conducted at 1 and 6 months recovery (i.e., after CSF1R inhibitor removal and myeloid cell repopulation). Sociability test: Crawley's or Three-Chamber Sociability test assesses general sociability, or time spent with another rodent. In brief, animals were placed in a ThreeChamber Sociability Test box $(19 \mathrm{~cm} \times 45 \mathrm{~cm})$ with two dividing walls made of clear Plexiglas allowing free access to each chamber. During habituation, the subject mouse is placed in the middle chamber for $5 \mathrm{~min}$ for adaption. During testing (24 h after habituation), a stranger mouse (inside a wire containment cup) is placed in one of the side chambers, and the subject mouse is placed in the center chamber and allowed to access and explore all three chambers for $10 \mathrm{~min}$. The placement of the stranger mouse in the left and right chambers is systemically altered between trials. The duration of time spent in each chamber, velocity, and distance traveled was measured. Spontaneous alternation $Y$-maze: For this task, mice were placed in a Y-maze $(35.2 \mathrm{~cm}$ arm length $\times 5 \mathrm{~cm}$ width $\times 20 \mathrm{~cm}$ sidewall height). Each animal was allowed to freely explore the arena for $8 \mathrm{~min}$. Distinct intra-maze visual cues were positioned at the end of each arm for spatial orientation. Spontaneous alternation, which measures the willingness of an animal to explore new environments, was measured by the number of triads, or entry of all three arms in a consecutive sequence (i.e., $\mathrm{ABC}$ and not $\mathrm{BAB}$ ). Contextual fear conditioning (CFC): For the training trial, mice were placed in a CFC chamber (Ugo Basile; $17 \mathrm{~cm}$ length $\times 17 \mathrm{~cm}$ width $\times 25 \mathrm{~cm}$ height) and allowed to explore for $2 \mathrm{~min}$. At $2 \mathrm{~min}$, the animal received one shock $(3 \mathrm{~s}, 0.5 \mathrm{~mA})$. Following the cessation of the shock, the animal remained in the chamber for an additional $30 \mathrm{~s}$ before being returned to their home cage. The testing trial was conducted $24 \mathrm{~h}$ later, in which the animal was placed in the chamber and allowed to explore for $5 \mathrm{~min}$. EthoVision Activity Analysis software was used to detect activity levels and freezing behaviors. Contextual memory was assessed by measuring freezing behaviors, defined as the total lack of body movement except for respiration. Unless otherwise indicated, behavioral readouts for all tasks were calculated from video using the EthoVision XT 14 tracking system (Noldus).

\section{Histology and confocal microscopy}

Fluorescent immunolabeling followed a standard indirect technique as described previously $[7,41]$. Free-floating brain sections were washed with $1 \mathrm{x}$ PBS, incubated in normal serum blocking solution (5\% normal serum $+0.2 \%$ Triton $\mathrm{X}-100$ in $1 \mathrm{x}$ PBS) for $1 \mathrm{hr}$ and then stained against primary antibodies overnight $(\sim 16-24 \mathrm{~h})$ at $4{ }^{\circ} \mathrm{C}$ in normal serum blocking solution utilizing the following primary antibodies and dilutions: ionized calcium binding adaptor molecule 1 (IBA1; 1:1000; 019-19741, Wako and ab5076, Abcam), P2RY12 (1:200; HPA014518, Sigma-Aldrich), TMEM119 (1: 200; ab209064, Novus), CD68 (1:1000; MCA1957GA, BioRad), AXL (1:100, AF854, R\&D Systems), Ki67 (1:200, ab16667, Abcam), glial fibrillary acidic protein (GFAP; 1: 3000, ab4674, Abcam), S100ß (1:200, ab52642, Abcam), microtubule-associated protein 2 (MAP 2; 1:500, ab32454, Abcam), NeuN (1:1000; MAB377, EMD Millipore), PSD95 (1:500, ab18258, Abcam), synaptic vesicle glycoprotein $2 \mathrm{~A}$ (SV2A; 1:200; 119 002, Synaptic Systems), doublecortin (DCX; 1:500; sc-8066, Santa Cruz Biotechnology), IgG (1: 
200, 12-371, Millipore), and fibrinogen (1:1000, A0080, Dako). Following this, sections were washed with $1 \mathrm{x}$ PBS, incubated in fluorescent dye (Alexa Fluor)-conjugated secondary antibodies (1:200 in normal serum blocking solution) for $1 \mathrm{~h}$ and then washed in 1x PBS and mounted on slides. Prussian blue staining was performed using an Iron Stain Kit (ab150674, Abcam) according to manufacturer's instructions with Nuclear Fast Red solution (N2030, SigmaAldrich). A spleen was used as a positive control illustrating how the protein-bound ferric iron pigment stains bright blue. High-resolution fluorescent images were obtained using a Leica TCS SPE-II confocal microscope and LAS-X software. Composite images were obtained by setting up zstacks ( $\times 20$ objective: $5 \mu \mathrm{m}$ thickness with $\sim 8$ sections; $\times$ 63 objective: $2 \mu \mathrm{m}$ thickness with $\sim 20$ sections) and then ultimately collapsed into an overlay image. Post image processing was done using LAS-X software. All adjustments were kept consistent between groups. For confocal imaging, one field of view (FOV) per brain region was captured per mouse unless otherwise indicated. For whole brain stitches, automated slide scanning was performed using a Zeiss AxioScan.Z1 equipped with a Colibri camera and Zen AxioScan 2.3 software. Microglial morphology was determined using the filament module in Bitplane Imaris 7.5, as described previously [16]. Cell quantities were determined using the spots module in Imaris. Percent coverage measurements were determined in ImageJ (NIH).

\section{RNA sequencing and analysis}

Total RNAs were extracted by using RNeasy Mini Kit (Qiagen). RNA integrity number (RIN) was measured, and samples with RIN $\geq 7.0$ were kept for library construction. cDNA synthesis, amplification, library construction, and sequencing were performed by Novogene Inc. using Illumina NovaSeq and HiSeq platforms with paired-end 150 bp (PE 150) sequencing strategy. Read alignment and expression quantification: Pair-end RNAseq reads were aligned using STAR v.2.5.1b with the options (--outFilterMismatchNmax 10 --outFilterMismatchNoverReadLmax 1 --outFilterMultimapNmax 10) [42]. Rsubread was used to generate feature counts [43]. Gene expression was measured using Limma, edgeR, and org.Mm.eg.db packages with expression values normalized as RPKM [44-47]. Differential expression analysis: Libraries with uniquely mapping percentages higher than $80 \%$ were considered to be of good quality and kept for downstream analysis. Protein coding and long non-coding RNA genes, with expression RPKM $\geq 1$ in at least three samples, were collected for subsequent analysis. Differential expression analysis was performed by using Limma, edgeR, and org.Mm.eg.db [44-47]. Differentially expressed genes (DEGs) were selected by using false discovery rate $($ FDR $)<0.05$. Top significant genes are displayed as a volcano plot constructed using
GLimma, ggplot2, and EnhancedVolcano (FDR $<0.05$, LogFC > 1) [48]. Normalized (min max normalization for each individual gene) log2-transformed expression values are displayed as a heatmap with hierarchical clustering utilizing pheatmaps. Weighted correlation network analysis: Network analysis was performed using weighted gene co-expression analysis (WGCNA) package in $\mathrm{R}$ [49]. First, bi-weighted mid-correlations were calculated for all gene pairs and then used to generate an eigengene network matrix, which reflects the similarity between genes according to their expression profiles. This matrix was then raised to power $\beta(\beta=18)$. Modules were defined using specific module cutting parameters (minimum module size $=100$ genes, deepSplit $=4$, and threshold of correlation $=0.2$ ). Modules with a correlation greater than 0.8 were merged. We used first principal component of the module, called signed bicor network, to correlate brain region, irradiation, and treatment. Hub genes were defined using intra-modular connectivity (kME) parameter of the WGCNA package. Gene enrichment analysis: Gene set enrichment analysis was done using enrichR package 71. Gene ontology and pathway analysis: Specific modules were analyzed for Gene ontology $(\mathrm{GO})$ enrichment by enrichR with corrected $p$ values $<0.05$. STRING interaction maps or functional protein association networks were generated using string-db.org (STRING Consortium 2020).

\section{Data analysis and statistics}

Statistical analysis was performed with Prism Graph Pad (v.8.0.1). To compare two groups, the unpaired Student's $t$ test was used. To compare multiple groups, a one-way ANOVA with Tukey's post hoc test was performed. For all analyses, statistical significance was accepted at $p<0.05$. All bar graphs are represented as means \pm SEM and significance expressed as follows: ${ }^{*} p<0.05,{ }^{* * *} p<0.01$, **** $p<$ $0.001 . n$ is given as the number of mice within each group.

\section{Results \\ Combining irradiation, bone marrow transplant, and microglial depletion achieves long-term and complete peripheral myeloid cell engraftment in the brain}

Here, we set out to explore as a proof-of-principle the clinical feasibility of a pharmacological-based method for near complete myeloid cell replacement. It is well known that BM-derived cells enter the brain following wholebody irradiation [50]; however, cell engraftment is limited. To this end, we and others [22] recently developed a unique paradigm, which utilizes whole-body irradiation, BM transplant, and a CSF1Ri treatment to achieve near complete parenchymal engraftment by donor peripheral myeloid cells (Fig. 1A-C). Previous studies have employed lead shielding of the head (i.e., body-only irradiation) to prevent peripheral-derived myeloid cell 
infiltration [22] and limit the effects of irradiation on the brain [51] caused during whole-body irradiation (i.e., head + body irradiation); however, we have found that head irradiation is required for optimal cell replacement. Although this method presents therapeutic potential for the replacement of dysfunctional myeloid cells, no studies to date have investigated the long-term consequences of such replacement. Given that microglia, nonparenchymal macrophages, and monocytes represent distinct heterogeneous populations of myeloid cells and it remains unclear whether these cells share overlapping functional capabilities, we set out to investigate the longterm consequences of microglial replacement with bone marrow (BM)-derived peripheral myeloid cells. To accomplish this, three experimental groups were generated: (1) WT CON, C57BL/6 wildtype (WT) control mice; (2) GFP-BM CON, WT mice subjected to whole body irradiation and retro-orbital infusion of donor CAG-green fluorescent protein (GFP) BM cells; and (3) GFP-BM REPOP, WT mice subjected to whole-body irradiation, GFP-BM transplant, and CSF1Ri-induced repopulation. It should be noted that WT CON received no irradiation, transplantation of GFP BM cells, or CSF1Ri treatment. Peripheral percent chimerism was evaluated after $\sim 12$ weeks via blood granulocytes and averaged $\sim 98.0 \%$ in both irradiated groups (Fig. 1G). Previous studies have shown that high-dose cranial irradiation increases BBB permeability, albeit transiently [52], and so animals were provided with a 3.5 months $\mathrm{BBB}$ recovery period prior to further treatment. After 3.5 months, mice were treated for 14 days with the CSF1Ri PLX3397 at a dose of $600 \mathrm{ppm}$ to deplete the microglial compartment. PLX3397 was then withdrawn, effectively stimulating myeloid cell repopulation. Under normal circumstances, repopulation of the microglial niche derives from the proliferation of surviving cells [7, 21, 53, 54], replacing the depleted tissue. However, in this study, we found that repopulation under exceptional conditions (i.e., following whole-body irradiation and microglial depletion) derives from BM-derived myeloid cells and results in the near complete replacement of the microglial niche with BM-derived peripheral myeloid cells (i.e., monocytes) within 14 days. These findings are in line with a previous study by Bruttger et al. showing that BM-derived macrophages repopulate the irradiated brain following genetic microglial depletion [54]. In their approach, Bruttger et al. utilize a genetic and toxinbased mouse model of microglial depletion (i.e., $C \times 3 \mathrm{cr}^{\mathrm{CreER}}{ }^{\text {iDTR }}$ system relying on tamoxifen and diptheria toxin administration), whereas we utilize CSF1R inhibitors to evaluate as a proof-of-principle whether replacement of microglia is a clinically feasible approach $[55,56]$. To explore the long-term consequences of this engraftment, animals remained on control chow for an additional 6 months prior to sacrifice ( 10 months after irradiation). At this time point, peripheral percent chimerism was evaluated via BMcollected hematopoietic stem cell (HSC) GFP expression and averaged $\sim 97 \%$ in both irradiated groups (Fig. 1H).

Immunohistochemical examination of the brains $\sim 10$ months after irradiation and $\sim 7$ months after PLX3397 treatment revealed the extent of BM-derived cell parenchymal engraftment. As expected, no GFP+ cells were found in the control brains (Fig. 1D). Irradiation alone, without microglial depletion/repopulation, caused partial engraftment of GFP+ BM-derived cells, particularly in meningeal and perivascular spaces, in line with prior reports (Fig. 1D; [57, 58]). Approximately $20 \%$ of parenchymal IBA1+ cells were GFP+, indicating that irradiation alone allows for limited access and engraftment of monocytes into the CNS (Fig. 1E). On the other hand, irradiated mice treated with CSF1Ri, allowing for myeloid cell repopulation (GFP-BM REPOP mice), displayed extensive $(\sim 80 \%)$ GFP+ myeloid cell chimerism, essentially replacing the entire microglial tissue with BM-derived cells (Fig. 1E-F). No differences in GFP+ myeloid cell repopulation were apparent between brain regions (Fig. 1D).

\section{Lasting effects of irradiation on BBB integrity, neurogenesis, and cell proliferation}

Prussian blue staining, which labels ferric iron, was performed to evaluate for the presence of microbleeds or microhemorrhages in the brain. No significant changes in Prussian blue were observed (Fig. 2A-B). Previous studies have shown that irradiation, with accompanying monocyte infiltration, has lasting effects on BBB integrity [52, 59-61]. Despite an observed influx of peripheralderived myeloid cells, BBB integrity was not compromised, as assessed by IgG, and fibrinogen staining $\sim 10$ months post-irradiation (Fig. 2C-D).

Another potent and long-term effect of irradiation involves impairing neurogenesis and cell proliferation [62, 63]. In line with these studies, we observe a significant decrease in doublecortin, a marker for neuronal precursor cells/neurogenesis, in the subgranular zone of the dentate gyrus in the hippocampus, a major site of neurogenesis in the mouse brain, following irradiation, which remains reduced following monocyte infiltration (Fig. 2E-H). Monocytes are short-lived cells, with a half-life of approximately 1-7 days in humans, whereas studies have shown that microglia exhibit much slower turnover rates with an average lifespan of 4.2 years in humans [64, 65]. To assess how the engrafted monocyte population is maintained over time, we explored cell proliferation using Ki67 and found no significant differences in IBA1+ cell proliferation. In fact, we detected no to little Ki67+IBA1+ cells in the cortex across all groups, including Ki67+GFP+ cells 


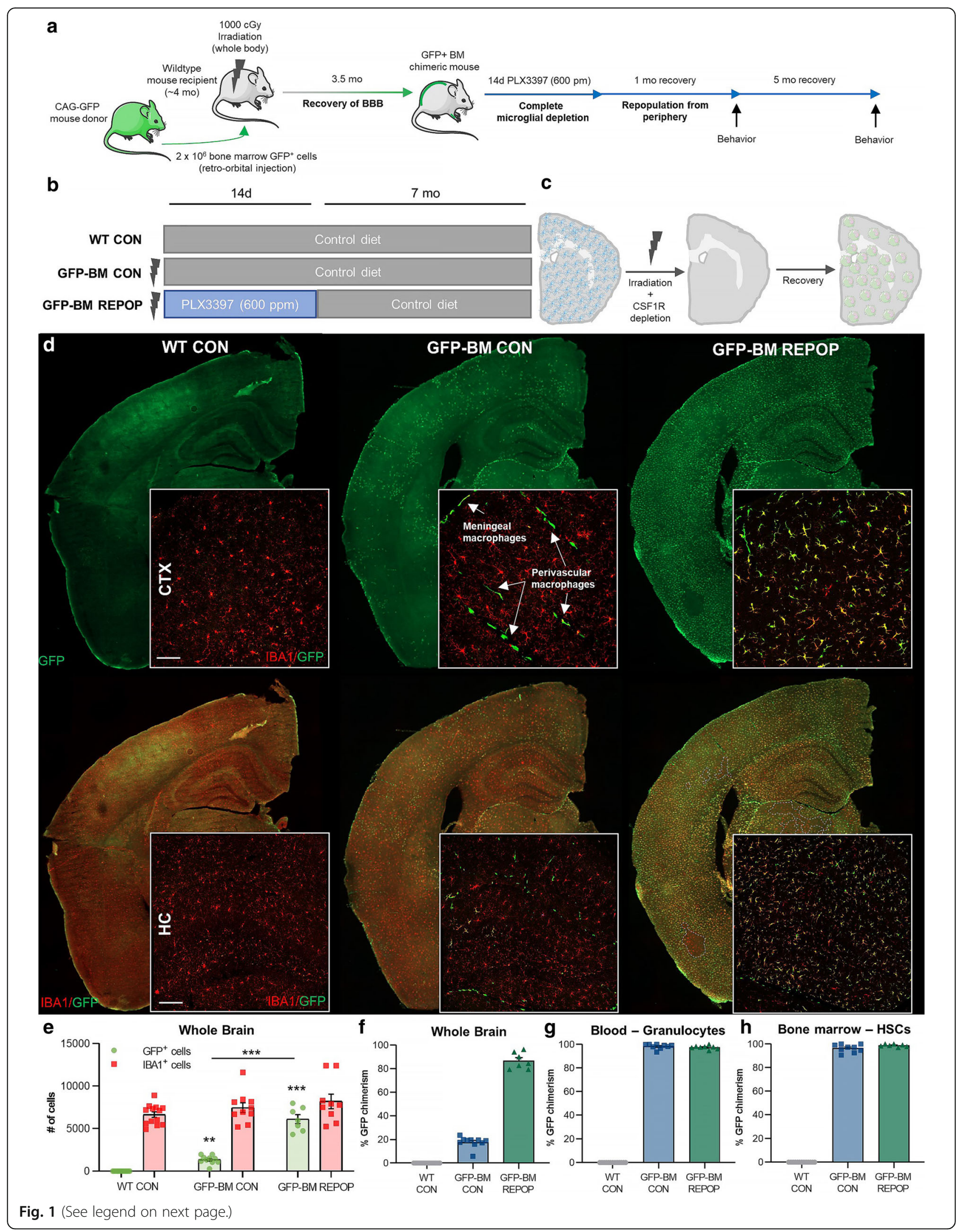




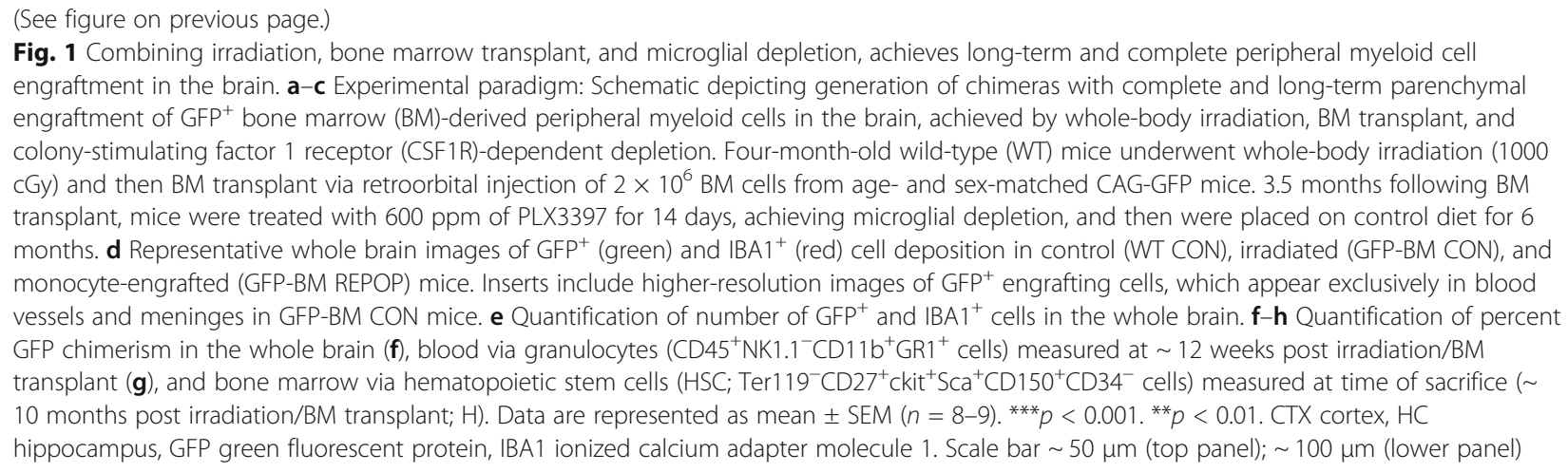

(Fig. 2I-J). In addition to examining these known longterm effects of irradiation, these data also suggest that once peripheral donor cells engraft the CNS, they are long-lived in the brain with very little cell turnover.

\section{Myeloid cell characterization after long-term peripheral myeloid cell engraftment}

Studies have shown that monocytes downregulate their canonical markers upon infiltration and differentiation in the CNS. Moreover, the loss of the homeostatic microglial signature (e.g., Sall1, Pu.1, Tmem119, Cx3cr1, and P2ry12) is associated with CNS disorders [66]; thus, we sought to characterize myeloid cell surface marker expression and morphology following irradiation and microglial replacement. Immunohistochemistry utilizing ionized binding adaptor molecule 1 (IBA1), a marker common to all myeloid cells, and P2RY12, a microglial specific marker, was performed on brains collected $\sim 7$ months following CSF1Ri-induced peripheral myeloid cell infiltration (Fig. 3A-D). Here, the remaining microglia form pockets between GFP+ spaces within the brain (Fig. 1D; Fig. 3A; outlined by white dotted lines; quantified in Fig. 3E-G). We define these pockets or islands of remaining microglia as areas of cells that are GFP- and often P2RY12+, which are only apparent in GFP-GM REPOP mice (Fig. 3A). GFP-BM REPOP mice also exhibited an elevated number of IBA1+ cells compared to controls (Fig. 3B-D and quantified in $3 \mathrm{H}$ ). Further characterization of IBA1+ cells shows that the number of IBA1+P2RY12+ cells is decreased in GFP-BM CON and GFP-BM REPOP mice (Fig. 3I), whereas the number of IBA1+GFP+ cells are only significantly increased in GFP-BM REPOP (Fig. 3J). These data indicate that the myeloid cells that fill the brain in GFP-BM REPOP mice are BM-derived cells and do not express a classical homeostatic microglial marker. IBA1+GFP+ BM-derived myeloid cells in GFP-BM REPOP mice also displayed distinct morphological differences from IBA1+ cells in WT CON and GFP-BM CON, as well as, IBA1+GFPcells in GFP-BM REPOP mice, in which they displayed smaller cell bodies (Fig. 3K), larger dendrites/cell processes (Fig. 3L), and reduced dendritic complexity (Fig. $3 \mathrm{M})$, consistent with BM-derived cells (i.e., monocytes) exhibiting more amoeboid-like morphology compared to microglia (refer to Fig. S1A for representative morphology reconstructions).

In addition, monocyte-engrafted brains displayed significantly increased CD68, a lysosomal marker associated with a heightened activation or phagocytic state, staining compared to controls and irradiated brains (Fig. 3N-O). As expected, all IBA1+ cells in control brains exhibited extensive TMEM119 immunoreactivity (Fig. 3P, S1B), and all GFP+ cells (i.e., BM/peripheral-derived cells) displayed little to no TMEM119+ staining (Fig. 3Q). In addition, it appears that irradiation induces a significant loss of TMEM119 expression (Fig. 3P, S1B). These data indicate that the engrafting BM-derived myeloid cells, even following 7 months of residence in the brain, continue to lack microglial signature expression. Collectively, these results show that although monocytes can take up residence in the brain, they maintain a unique cell surface marker and morphological identity within the brain parenchyma, even after extended periods of residence in the brain.

After evaluating all IBA1+ cells, we next sought to delineate infiltrating monocytes from microglia unaltered by treatment, referred to as remaining microglia. Analysis of images in regions of extensive infiltrating monocyte engraftment or extensive remaining microglia presence shows that the loss in the microglial homeostatic signature (i.e., TMEM119) is specific to monocytes. Expression of TMEM119 in remaining microglia is not significantly altered by irradiation or repopulation (Fig. 3Q-R). In addition, we also analyzed AXL, a protein enriched in phagocytic cells, and observe significant increased expression of AXL in infiltrating monocytes in both irradiated groups, as well as, remaining microglia in GFP-BM REPOP. However, AXL expression appears most elevated in infiltrating monocytes in GFP-BM REPOP mice (Fig. 3S-T). 


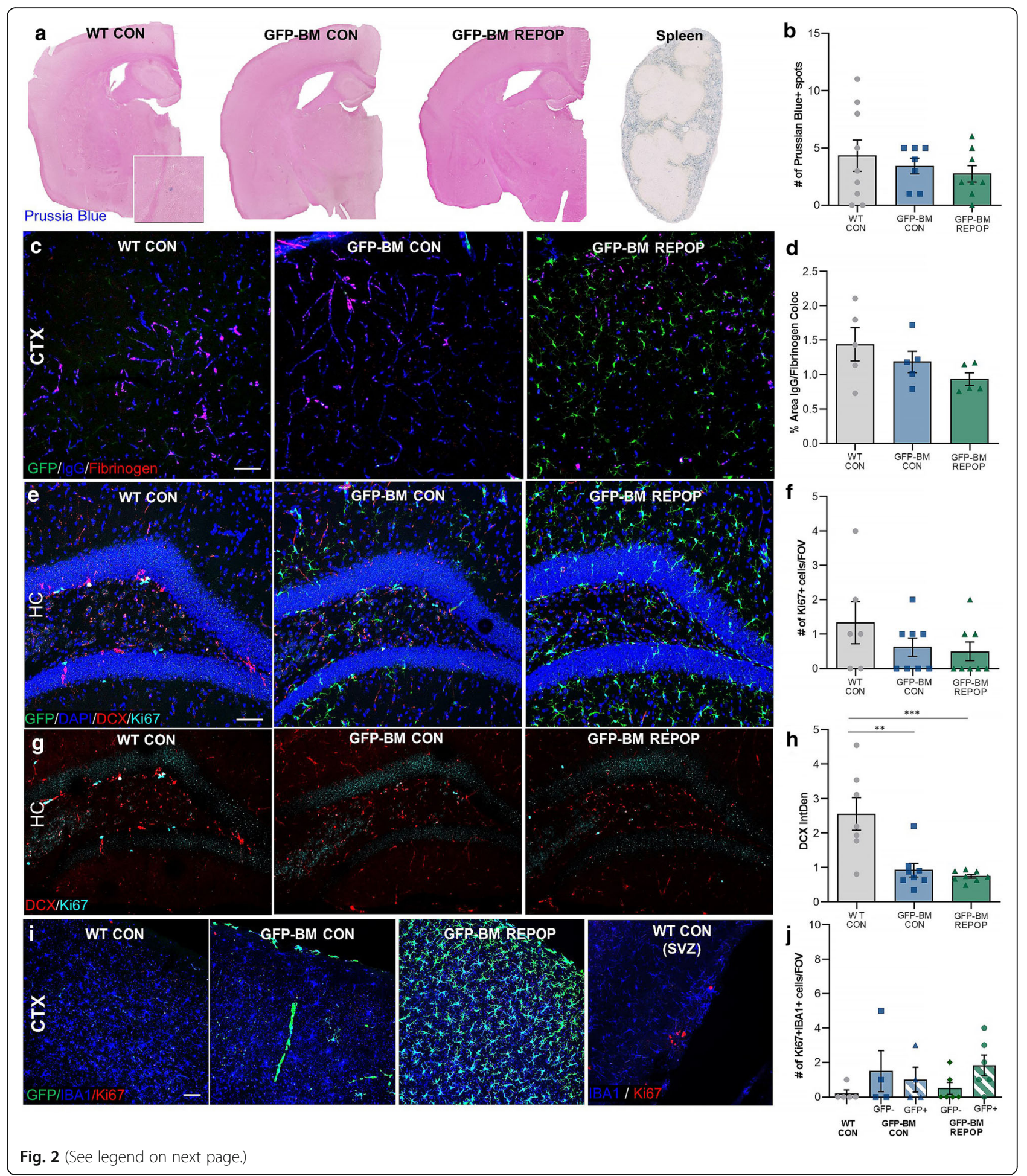




\section{(See figure on previous page.)}

Fig. 2 Lasting effects of irradiation on BBB integrity, neurogenesis, and cell proliferation. a Representative whole brain images of blood-brain barrier (BBB) integrity via Prussian blue (blue) in control (WT CON), irradiated (GFP-BM CON), and monocyte-engrafted (GFP-BM REPOP) mice. Spleen provides as a positive control. Insert shows higher-resolution images of a Prussian blue ${ }^{+}$spot. $\mathbf{b}$ Quantification of number of Prussian Blue $^{+}$spots in the whole brain. c Representative immunofluorescence images of immunoglobulin (lgG, blue), fibrinogen (red), and GFP (BMderived cells, green) in the cortex. d Quantification of \% area of lgG and fibrinogen colocalization in the cortex. e Representative immunofluorescence images of neurogenesis marker doublecortin ${ }^{+}$(DCX, red), proliferation marker Ki67 $7^{+}$(light blue), DAPI ${ }^{+}$(blue), and GFP ${ }^{+}$ (green) staining. $\mathbf{f}$ Quantification of Ki67 $7^{+}$cells per FOV in the dentate gyrus of the hippocampus. $\mathbf{g}$ Representative immunofluorescence images of neurogenesis marker doublecortin ${ }^{+}$(DCX, red) and proliferation marker Ki67 $7^{+}$(light blue) staining. $\mathbf{h}$ Quantification of DCX staining intensity in the dentate gyrus of the hippocampus. i Representative immunofluorescence images of proliferating myeloid cells stained for IBA1 (blue), proliferation marker Ki67 (red), and GFP (green). j Quantification of $\mathrm{Ki}_{6} 7^{+} \mid \mathrm{BA} 1^{+}$(including $\mathrm{GFP}^{+}$and $\mathrm{GFP}^{-}$) cells per FOV. Data are represented as mean \pm SEM $(n=5-8) .{ }^{* *} p<0.01,{ }^{* *} p<0.001$. CTX cortex, HC hippocampus, SVZ subventricular zone, GFP green fluorescent protein, IBA1 ionized calcium adapter molecule 1. Scale bar $\sim 50 \mu \mathrm{m}$

\section{Transcriptional changes following long-term peripheral myeloid cell engraftment}

After detecting alterations in myeloid cell expression, we continued our investigations by addressing the impact of long-term peripheral myeloid cell engraftment at the transcriptional level. Since previous studies have shown that microglial heterogeneity is detected in a distinct brain region-specific manner by bulk tissue RNA sequencing (RNAseq) [67-70], we performed RNAseq analysis on three brain regions (cortex, hippocampus, and thalamus/ striatum). Gene expression can be explored at http://rnaseq.mind.uci.edu/green/long-term_monocytes/. To initially identify gene expression changes due to both irradiation and the brain-wide presence of monocytes, we compared the transcriptomes of GFP-BM REPOP mice to controls (WT CON), which resulted in 2695 differentially expressed genes (DEGs; FDR < 0.05 ) across all brain regions. Notably, the effects were most prominent in the thalamus (2401 DEGs), followed by the hippocampus (370 DEGs), with few DEGs detected in the cortex (36 DEGs; Fig. 4A). We next examined transcriptional changes due to irradiation alone (i.e., WT-CON vs. GFP-BM CON), and due to the presence of monocytes (i.e., GFP-BM CON vs. GFP-BM REPOP; Fig. 4A) and display the results as volcano plots (Fig. 4B-D). Separating the effects of irradiation and monocyte infiltration, we observe that the hippocampus and thalamus are more vulnerable to irradiation-induced gene changes, compared to the cortex, which shows little to no changes. Of note, the most significant transcriptional changes due to monocyte infiltration occur exclusively in the hippocampus. Overall, these results demonstrate that different brain regions exhibit selective vulnerabilities to irradiation and the infiltration of monocytes. Using the DEGs present in the transcriptional comparison between WT CON vs. GFP-BM REPOP mice across all three brain regions, we were able to build a unique long-term monocyte-related signature (Fig. 4E). Among these genes most prominently upregulated in monocyte-engrafted mice, we identified Apobec1, the chemokine receptor $C c r 1$, the C-type lectin $M r c 1$, and several members of the Ms4a cluster (Ms4a6b, Ms4a6c, and
Ms4a7). In this comparison, known microglial specific genes were also downregulated, including Crybb1, Slc2a5, Siglech (selected genes shown in Fig. S2A).

To understand and place transcriptional changes associated with microglial-monocyte replacement and irradiation within three distinct brain regions on a broader system-level scale, we next conducted weighted gene coexpression network analysis (WGCNA) [49]. Twentytwo distinct modules were identified and given colorbased names (Fig. 4F). The correlation between modules is shown in Fig. S2B, highlighting modules that behave similarly to one another. We initially focused on modules that exhibited the highest correlation with monocyte presence in the brain ("treatment" variable) and identified a single module: darkgreen (Fig. 4G-I). Eigengene values for darkgreen showed consistent increases in GFP-BM REPOP mice across all three brain regions, reflected in the provided heatmap (Fig. 4H). Furthermore, cell-type enrichment analysis (Fig. 4O) demonstrated that darkgreen genes are myeloid-enriched and included previously identified peripheral myeloid-related genes: Apobec1, Apoe, Clec7a, Ms4a6c, and Ms4a7. Accordingly, pathway analyses listed the top Biological Process Gene-Ontology (GO) terms: response to other organism, defense response to protozoan, response to external stimulus, defense response, and positive regulation of cytokine production. Genes in the darkgreen module are displayed in a functional protein-protein interaction network highlighting genes (in red) in defense response (Fig. 3I). In addition to identifying a monocyte-specific signature module, we also identified several modules associated with irradiation across all three brain regions. Here, we provide one module: salmon (Fig. 4J-K). Celltype enrichment analysis shows that the salmon module is significantly enriched with interneuron genes (Fig. 4O). Functional annotation of the salmon module shows that it is enriched in the following top GO terms: cellular process and gene expression, and Reactome Pathways: Metabolism of RNA, Processing of Capped intronContaining Pre-mRNA, mRNA Splicing, and RNA Polymerase II Transcription. These findings are consistent 


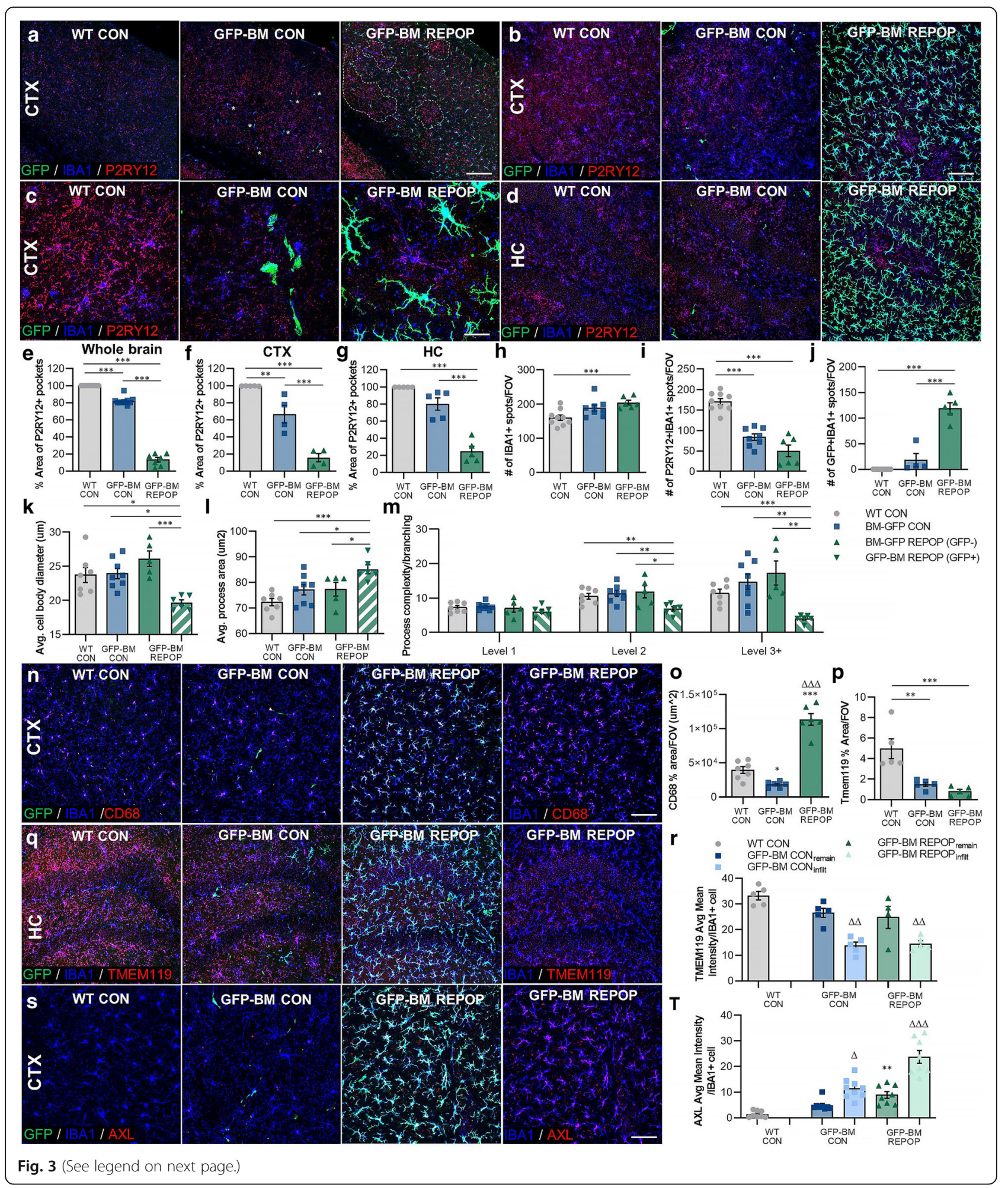


(See figure on previous page.)

Fig. 3 Myeloid cell characterization after long-term peripheral myeloid cell engraftment. a-d Representative immunofluorescence images of myeloid cells in the brains of control (WT CON), irradiated (GFP-BM CON), and monocyte-engrafted (GFP-BM REPOP) mice stained for common myeloid marker ionized calcium-binding adaptor molecule 1 (IBA1, blue), microglial specific marker P2RY12 (red), and GFP (BM-derived cells, green) in the cortex $(\mathbf{a}-\mathbf{c})$ and hippocampus (d). Higher-resolution image showing P2RY12 immunoreactivity of IBA $1^{+}$and $\mathrm{GFP}^{+}$cells $(\mathbf{c})$. White dotted areas indicate pockets of high P2RY12 immunoreactivity, which coincide with a lack GFP ${ }^{+}$cells. e-m Quantification of P2RY12, IBA1, and $\mathrm{GFP}^{+}$staining: \% area of $\mathrm{P} 2 \mathrm{RY} 12^{+}$pockets, defined also as areas lacking GFP ${ }^{+}$cell infiltrates $(\mathbf{e}-\mathbf{g}), \mathrm{IBA} 1^{+}$cell number per field of view (FOV) (h), P2RY $12^{+} \mid \mathrm{BA} 1^{+}$cell number per field of view (FOV) (i), GFP $\mid \mathrm{BA} 1^{+}$cell number per field of view (FOV) (j). For these measurements, images were captured and quantified in the hippocampus. $\mathbf{k}-\mathbf{m}$ Quantification of IBA ${ }^{+}$cell morphology: average cell body diameter size (k), average process area $(\mathbf{I})$, and process branching complexity $(\mathbf{m})$. For these measurements, images were captured and quantified in the hippocampus. GFP ${ }^{+}$ staining was also used to distinguish between remaining microglia $\left(\mathrm{IBA} 1^{+} \mathrm{GFP} \mathrm{P}^{-}\right.$) and infiltrating monocyte $\left(\mathrm{IBA} 1^{+} \mathrm{GFP}{ }^{+}\right.$) morphologies in GFP-BM REPOP mice. $\mathbf{n}$ Representative immunofluorescence images of myeloid cells stained for IBA1 (blue), macrophage/monocyte marker CD68 (red), and GFP (green). o Quantification of CD68 staining area coverage per FOV. $\mathbf{p}$ Quantification of TMEM119 staining intensity in the hippocampus. $\mathbf{q}$ Representative immunofluorescence images of myeloid cells stained for IBA1 (blue), CNS myeloid cell-specific marker TMEM119 (red), and GFP (green) in the hippocampus. $\mathbf{r}$ Quantification of the average mean intensity of TMEM119 in $\mid B A 1^{+}$cells per FOV, comparing remaining microglia (remain) and infiltrating BM-derived cells (infilt). s Representative immunofluorescence images of proliferating myeloid cells stained for IBA1 (blue), phagocytic marker AXL (red), and GFP (green). $\mathbf{t}$ Quantification of the average mean intensity of AXL in $\mid B A 1^{+}$cells per FOV, comparing remaining microglia (remain) and infiltrating BM-derived cells (infilt). Data are represented as mean \pm SEM $(n=4-9) .{ }^{*} p<0.05,{ }^{* *} p<0.01,{ }^{* * *} p<0.001$. CTX cortex, HC hippocampus, GFP green fluorescent protein, IBA1 ionized calcium adapter molecule 1. Scale bar $\sim 150 \mu \mathrm{m}(\mathbf{a}) ; \sim 75 \mu \mathrm{m}(\mathbf{b}, \mathbf{d}, \mathbf{n}, \mathbf{q}) ; \sim$ $60 \mu \mathrm{m}(\mathbf{s}) ; \sim 25 \mu \mathrm{m}(\mathbf{c})$

with the known effects of irradiation, including DNA damage and repair [71], and recent studies have shown that splicing factors are recruited to sites of DNA damage [72]. Several modules indicate that monocyte engraftment leads to a partial recovery of irradiation-induced effects, specifically in the hippocampus. Here, we show one module: brown (Fig. 4L-N). Cell enrichment analysis revealed the brown module to be highly enriched for astrocyte-expressed (astro) genes (Fig. 4O). The top GO terms identified in this module were cilium organization, plasma membrane bounded cell projection assembly, cilium assembly, and cell projection organization. Genes in the brown module are displayed in a functional protein-protein interaction network highlighting genes (in red) in cilium organization (Fig. $4 \mathrm{~N})$. Cilia are small microtubulebased signaling projections expressed on virtually all cells, including neurons and glia. Cilium function is implicated in sensing the extracellular environment, signal transduction, and regulating cell division/cell cycle [73]. Recent studies also indicate that cilia and cilia-associated proteins may play a role in neuronal development and DNA damage and repair [74]. Together, these data indicate that irradiation and the presence of repopulating monocytes play a role in altering astrocyte and neuronal-related genes in the brain, as well as, exhibit distinct effects in different brain regions, including the cortex and hippocampus.

\section{The differential and brain region-dependent effects of long-term peripheral myeloid cell engraftment on astrocytic and neuronal properties}

To further explore these differential effects, we next assessed astrocytes and neurons in various regions of the brain using immunohistochemical analysis. Astrocytes, the other major glial cell of the CNS, were stained using astrocyte markers glial fibrillary acidic protein (GFAP) and S100 $\beta$ (Fig. 5A-C; Fig. S3A, B). S100 $\beta+$ (Fig. 5D; Fig. S3A) and GFAP+ (Fig. 5F; Fig. S3B) cells were significantly elevated in the cortex of GFP-BM REPOP mice. A significant elevation in $\mathrm{S} 100 \beta+$ cells was also found in hippocampus in these mice (Fig. 5E), but no differences were detected in the number of GFAP+ cells (Fig. 5G), indicating that monocyte-engrafted brains show heightened astrocyte numbers for specific astrocyte subsets in a brain region-dependent manner. Furthermore, we observed a significant increase in GFAP+ staining intensity in the hippocampus of BM-GFP CON mice (Fig. $5 \mathrm{H}$ ), indicating that astrocytes in the hippocampus of irradiated mice are not elevated in cell number, but appear more activated, and that this activation is reversed by monocyte engraftment. These data are in line with transcriptional findings that monocyte engraftment reverses irradiation-induced astrocyte-associated gene changes (Fig. 4L-N).

In addition to astrocyte alterations, we also explored neurons and associated structural properties (e.g., axons and synapses). No gross differences were detected in neuronal numbers following irradiation or long-term monocyte engraftment, as assessed by neuronal marker NeuN (Fig. 5I-J). However, as has been previously reported by others, microtubule-associated protein 2 (MAP2) was significantly decreased in both brain regions (i.e., cortex, hippocampus) in GFP-BM CON mice (Fig. 5K, N-O) [75-77]. Consistent with previous findings in the hippocampus, a significant reduction in MAP2 expression was not seen in GFP-BM REPOP mice, indicating a reversal in irradiation-induced changes following monocyte 


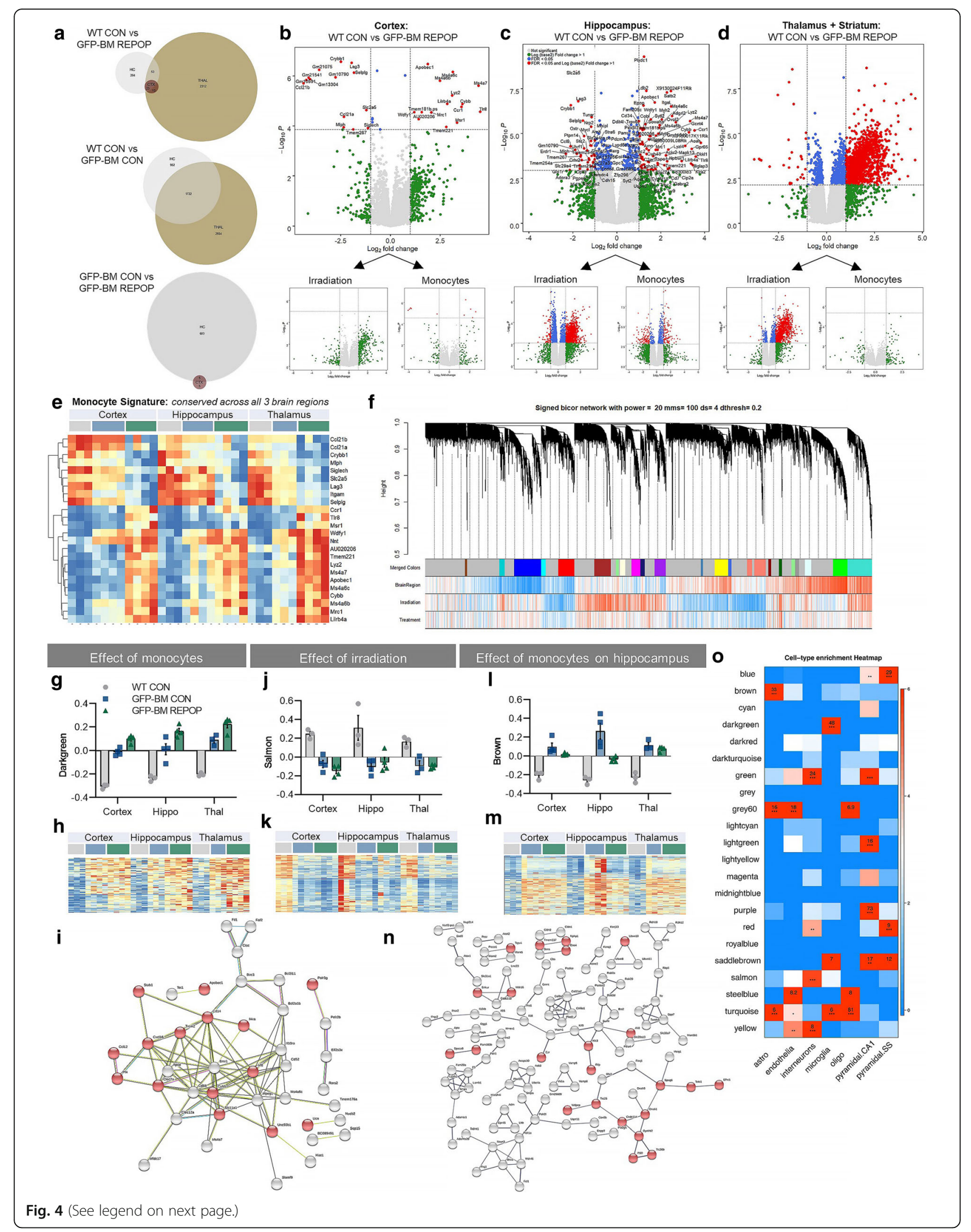




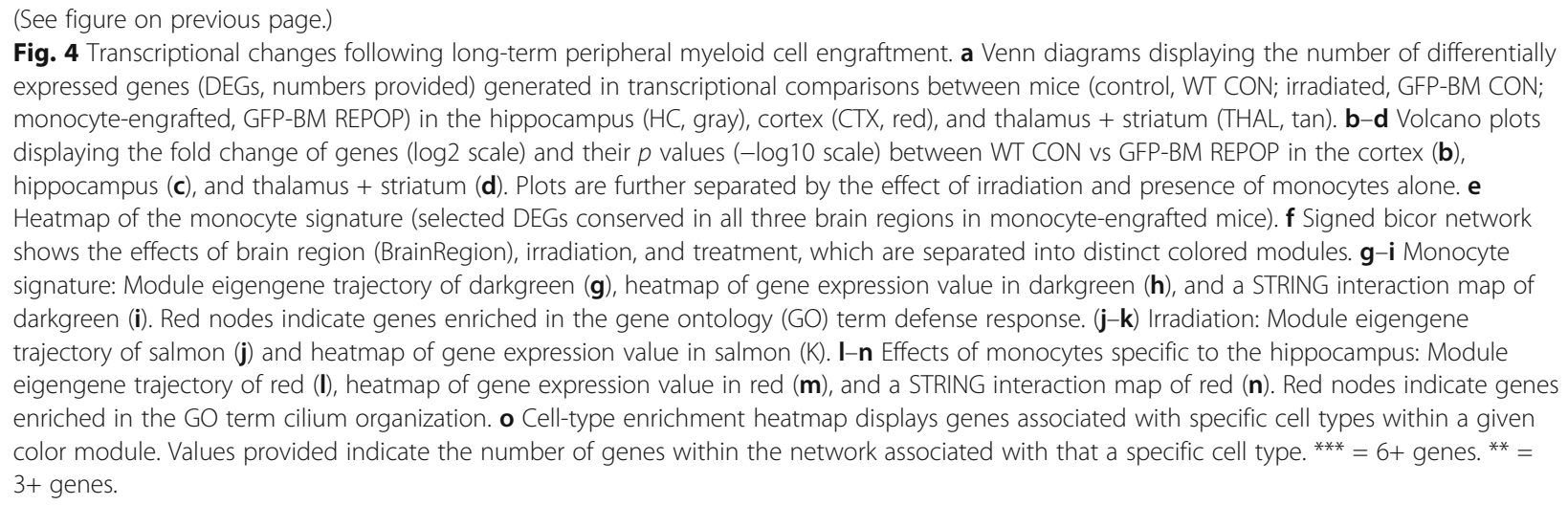

engraftment. Next, we explored synaptic alterations using postsynaptic density 95 (PSD-95; Fig. 5L-L'), a major scaffolding protein expressed on excitatory synapses, and synaptic vesicle glycoprotein 2A (SV2A; Fig. 5M), a synaptic vesicle protein expressed ubiquitously in the CNS present on GABAergic and glutamatergic presynaptic terminals [78]. A significant loss of PSD95+ synapses was found in GFP-BM REPOP mice in the cortex, and a significant loss was found in GFP-BM CON mice in the hippocampus but not in GFP-BM REPOP mice (Fig. 5L-L', P-Q). No significant alterations in SV2A staining were detected (Fig. $5 \mathrm{M}, \mathrm{R}-\mathrm{S})$. Together, these data indicate that irradiation and monocyte engraftment can have differential effects on astrocytic and neuronal properties in a brain regiondependent manner. These data also confirm that in the hippocampus, monocyte engraftment appears to reverse some irradiation-induced effects.

\section{Effects of long-term peripheral myeloid cell engraftment on behavior and cognition}

After observing changes at the cellular level, we next sought to determine whether irradiation and/or myeloid cell replacement leads to short- or long-term alterations in behavior or cognition. To accomplish this, mice underwent behavioral and cognitive testing 1 and 6 months following CSF1Ri-induced repopulation (Fig. 1A). Mice were first tested on the elevated plus maze (Fig. 6A-D). Neither irradiated (GFP-BM CON) nor repopulated (GFP-BM REPOP) mice exhibited changes in anxiety during this task at 1 month (Fig. 6B) or 6 months (Fig. 6D) post-repopulation compared to controls (WT CON), and no motor differences were recorded during this task (Fig. 6A, C). Despite the lack of changes in the elevated plus maze, we did observe significant differences during open-field analyses in distance traveled (Fig. $6 \mathrm{E}$ ) in both transplanted groups compared to WT CON at 1 month, showing that irradiation impacts locomotion. However, these significant alterations were transient and not detected at 6 months (Fig. 6H). Evaluations of locomotive activity utilizing a rotarod apparatus show that irradiation does slightly impact average motor function over all trials $(p=0.055)$ at 1 month (Fig. 6K). GFP-BM $\mathrm{CON}$ mice improve over time and stay on longer compared to controls (Fig. 6L); however again, these alterations were transient and not detected at 6 months (Fig. $6 \mathrm{M}-\mathrm{N})$. To assess recognition memory, mice were tested using the novel place recognition test. In this task, we observed no significant differences between groups (Fig. 6FG, I-J); however, we do observe a slight increase in memory as measured by preference index in GFP-BM REPOP mice at 1 month. To further investigate these memory alterations, hippocampal-dependent learning and memory was assessed using the contextual fear conditioning apparatus (Fig. 6O-R). No significant differences were detected between groups as measured by \% freezing at 1 month (Fig. 6O-P) and 6 months (Fig. 6Q-R), indicating that hippocampal learning and memory is not altered by irradiation or the presence of monocytes. Additional behavior and cognitive testing were added at 6 months, including Crawley's sociability test (Fig. 6S-T) and spontaneous alternation Y-maze (Fig. 6U-W). No significant differences in general sociability were found between groups, as assessed by time spent in empty and stranger mouse-filled chambers (Fig. 6T). The spontaneous alternation Y-maze serves as an index for active retrograde working memory, which relies on the proper functioning of many brain regions, including the hippocampus and cortex [79]. Here, we observed the most overt behavioral alterations as a result of irradiation. Both GFP-BM CON and GFP-BM REPOP exhibited significantly reduced zone alternation frequency compared to WT CON (Fig. 6W), indicating that working memory may be impaired by irradiation. It should be noted that the number of total arm entries was slightly reduced in GFP-BM REPOP mice (Fig. 6V). Together, these findings indicate that by most behavioral tests, irradiation and monocyte engraftment result in little changes to behavior and cognition; however, there appears to be a significant long-term deficit in retrograde working 


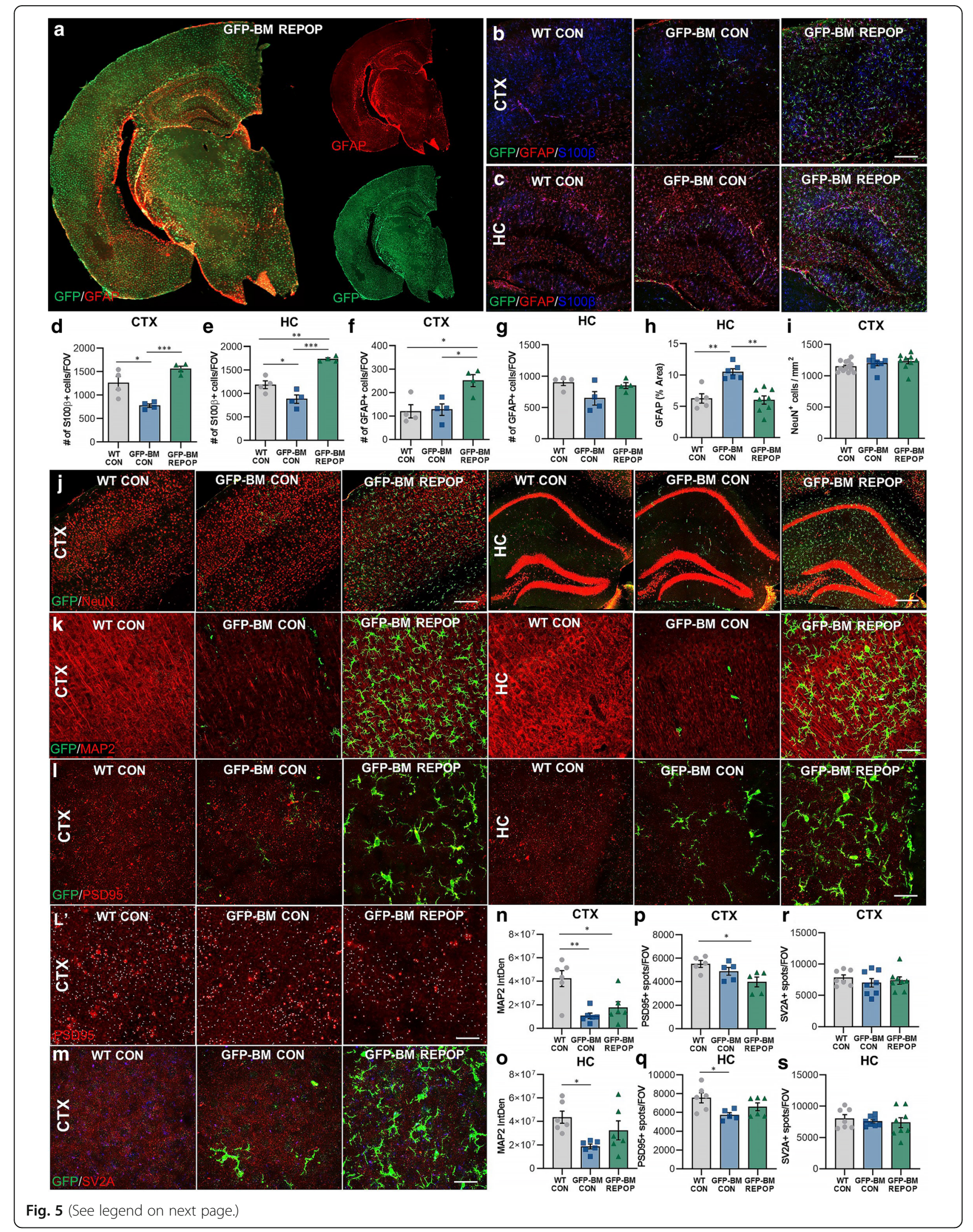




\section{(See figure on previous page.)}

Fig. 5 The differential and brain region-dependent effects of long-term peripheral myeloid cell engraftment on astrocytic and neuronal properties. a Representative whole brain images of GFP (green) and common astrocyte marker glial fibrillary acidic protein (GFAP, red) cell deposition in monocyte-engrafted mice. $\mathbf{b}-\mathbf{c}$ Representative immunofluorescence images of different astrocyte subtypes stained for GFAP (red) and S100ß (blue) in the in the cortex (B) and hippocampus (C) of control (WT CON), irradiated (GFP-BM CON), and monocyte-engrafted (GFP-BM REPOP) mice. GFP (green) stains BM-derived cells. $\mathbf{d}-\mathbf{g}$ Quantification of $S 100 \beta^{+}$and $G F A P^{+}$cell number in the cortex (D, F) and cell number in the hippocampus $(\mathbf{e}, \mathbf{g}) \cdot \mathbf{h}$ Quantification of GFAP staining intensity in the hippocampus. (i) Quantification of NeuN ${ }^{+}$cell number in the whole cortex. (j) Representative immunofluorescence images stained for neuronal marker NeuN (red) and GFP (green, BM-derived cells) in the cortex and hippocampus. (k) Representative immunofluorescence images of microtubule associated protein 2 (MAP2, red) and GFP (green, BM-derived cells) in the cortex and hippocampus. (I) Representative immunofluorescence brain images of synaptic marker PSD95 (postsynaptic density protein 95, red) and GFP (green, BM-derived cells) in the cortex and hippocampus. L' provides representative images of spots modeling using Imaris spots module software overlaid on PSD95 ${ }^{+}$staining. (m) Representative immunofluorescence brain images of synaptic marker SV2A (synaptic vesicle glycoprotein 2A, red) and GFP (green, BM-derived cells) in the cortex. (n-s) Quantification of the integrated density of MAP2 staining in the cortex (n) and hippocampus (o), total number of PSD95 $5^{+}$spots per field of view in the cortex (p) and hippocampus (q), and total number of SV2A ${ }^{+}$spots per field of view in the cortex $(\mathbf{r})$ and hippocampus $(\mathbf{s})$. Data are represented as mean \pm SEM $(n=4-9) .{ }^{*} p<0.05$, ${ }^{* *} p<$ $0.01 .{ }^{* * *} p<0.001$. CTX cortex, HC hippocampus, GFP green fluorescent protein, IBA1 ionized calcium adapter molecule 1. Scale bar $\sim 150 \mu \mathrm{m}$ (BC, j-left panel); $200 \mu \mathrm{m}$ (j-right panel); $40 \mu \mathrm{m}(\mathbf{k}) ; \sim 30 \mu \mathrm{m}(\mathbf{I}, \mathbf{m}) \sim 15 \mu \mathrm{m}\left(\mathrm{L}^{\prime}\right)$

memory, which is associated with the temporal lobe and prefrontal cortex.

\section{Discussion}

Monocytes derive from myeloid precursor cells in the $\mathrm{BM}$ and possess the ability to differentiate into tissuespecific macrophages in various organs and tissues, including the CNS. However, the role of these bloodderived myeloid cells in the CNS along with their ability to fill the microglial niche and long-term effects on the brain remain unclear. Here, we developed a paradigm using whole body irradiation, BM transplant, and pharmacological inhibition of CSF1R to achieve near complete and long-term engraftment of peripheral derived myeloid cells (i.e., monocytes) in the brain. Previous studies have shown that the microglial population relies on CSF1R signaling for survival and, thus, can be eliminated and repopulated using CSF1Ri administration and withdrawal, respectively $[7,16,18,21,53,80]$. Here, our data show that CSF1R inhibition following whole body irradiation results in the near complete $(\sim 80 \%)$ replacement of microglia with monocytes. These data indicate that monocytes are capable of outcompeting endogenous CSF1Ri-resistant microglia under these conditions. Previous data has shown that microglia survive cranial irradiation, however do exhibit increased activation and decreased cell number [81], and that irradiation induces loss of proliferating cells, including progenitor/ stem and microglial cells [60]. Thus, in order to induce complete monocyte engraftment, it appears that the brain must possess an empty microglial niche or microglial cells that lack the ability to proliferate/self-renew. In line with this, Bruttger et al. showed that genetic microglial ablation of BM chimeric mice (i.e., exposed to irradiation) resulted in repopulation of the microglial pool with BM-derived myeloid cells [54]. Furthermore, recent findings have demonstrated that chronic microglial depletion, using a genetic model of partial microglia deficiency, or CSF1Ri treatment (during irradiation) leads to monocyte engraftment in the CNS $[22,82]$.

Recent data demonstrate that monocytes are capable of filling the microglial niche [83] and taking up residence in the brain; however, these peripheral-derived cells remain phenotypically, transcriptionally, epigenetically, and functionally distinct from their microglial counterparts $[22,24$, $82,84,85]$. In this study, we observe that engrafted monocytes maintain a loss of homeostatic microglial signature markers and an increase in markers associated with phagocytosis. To address whether these differences translate into long-term consequences in the brain, we evaluated mice for transcriptional, cellular, and behavioral changes 6 months following monocyte engraftment into the CNS.

Transcriptional analysis reveals that the thalamus and hippocampus are the most vulnerable brain regions to irradiation. Further stratification of these comparisons teasing apart irradiation and monocyte-specific effects shows that the hippocampus is the only brain region that exhibits significant translational changes in response to monocyte infiltration. Notably, WGCNA analysis identified several modules elucidating a reversal in irradiation-induced effects following monocyte engraftment, occurring exclusively in the hippocampus. Pathway analysis of these modules reveals mechanisms involving cilium organization and assembly. A recent study using granulocyte colony-stimulating factor (GCSF) receptor knockout mice and BM transplant show that BM cells (responding to G-CSF) home to the irradiated brain and promote brain repair and neural progenitor cell proliferation [86]. Recent reports have demonstrated that brain-engrafted monocytes exhibit a distinct gene signature compared to microglia [22, 82]. Here, we highlight a distinct gene signature of engrafted monocytes compared to microglia following 6 months of recovery from CSF1Ri administration and 10 months following irradiation. Our monocyte signature includes an 


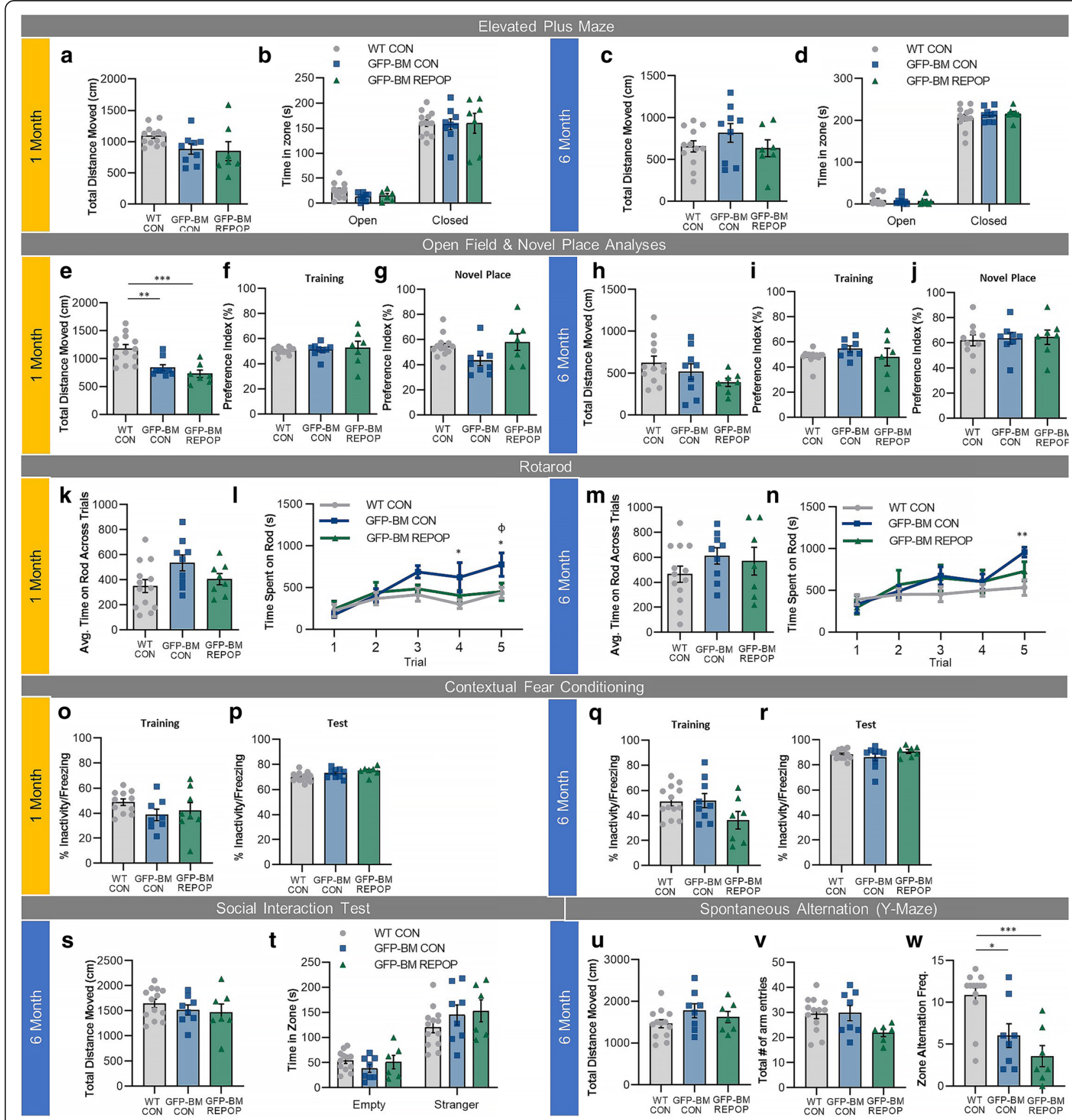

Fig. 6 Effects of long-term peripheral myeloid cell engraftment on behavior and cognition. (a-d) Quantification of elevated plus maze performance at 1 month (a-b) and 6 months ( $\mathbf{c}-\mathbf{d}$ ) post CSF1Ri treatment in control (WT CON), irradiated (GFP-BM CON), and monocyte-engrafted (GFP-BM REPOP) mice as assessed by time spent in open and closed arms (b, d). Total distance moved was also measured to control for locomotive changes $(\mathbf{a}, \mathbf{c})$. (e-j) Quantification of open field $(\mathbf{e}, \mathbf{h})$ and novel place recognition $(\mathbf{g}, \mathbf{j})$ performance at 1 month $(\mathbf{e}-\mathbf{g})$ and 6 months $(\mathbf{h}-\mathbf{j})$ as assessed by total distance moved $(\mathbf{e}, \mathbf{h})$ and percent preference index $(\mathbf{f}-\mathbf{g}, \mathbf{i}-\mathbf{j})$, respectively. Preference index is the ratio of the amount of time spent exploring any one of two objects $(A$ or $B)$ in the training phase or the novel-placed object $(C)$ in the test phase over the total time spent exploring both objects, i.e. $C /(B+C) \times$ 100 in the test phase. Quantification of training also provided $(\mathbf{f}, \mathbf{i})$. ( $(\mathbf{k}-\mathbf{n})$ Quantification of rotarod performance at 1 months $(\mathbf{k}-\mathbf{l})$ and 6 months $(\mathbf{m}-\mathbf{n})$. (o-r) Quantification of contextual fear conditioning at 1 months (o- $\mathbf{p})$ and 6 months (q-r) post CSF1Ri-induced monocyte engraftment as assessed by percentage of inactivity at testing $(\mathbf{p}, \mathbf{r})$. Quantification of training also provided $(\mathbf{o}, \mathbf{q})$. $\mathbf{s}-\mathbf{t}$ Quantification of social interaction test performance at 6 months post CSF1Ri treatment as assessed by time spent in an empty zone or zone containing a stranger mouse (t). Total distance moved was also measured to control for locomotive changes (s). $\mathbf{u}-\mathbf{- w}$ Quantification of spontaneous alternation (Y-maze) performance at 6 months post CSF1Ri treatment as assessed by zone alternation frequency $(\mathbf{w})$. Total distance moved and total number of arm entries was also measured to control for locomotive changes $(\mathbf{u})$ and motivation/movement $(\mathbf{v})$, respectively. ${ }^{*} p<0.05 .{ }^{* * *} p<0.001$ 
upregulation in Ccr1, Ms4a6b, Ms4a6c, Ms4a7, AU020206, Apobec1, Lyz2, Mrc1, Tmem221, Tlr8, Lilrb4a, Msr1, Nnt, and Wdfy1, coinciding with a downregulation of Siglech, Itgam, Selpig, Lag3, Slc2a5, Mlph, Crybb1, Ccl21a, and $C c l 21 b$. Notably, this differential gene signature was not detected in brain-extracted monocytes [22], except for an overlap in expression of Tlr8 between the two datasets, showing how extraction techniques and/or time in the brain play critical roles in monocyte gene expression (i.e., 6 weeks vs 6 months). Tlr8 is a member of the toll-like receptor family and plays an important role in pathogen recognition and innate immunity. It is also a gene that is predominantly expressed on peripheral blood leukocytes.

On the cellular level, brain region-specific differences in irradiation and monocyte engraftment were apparent. Astrocyte numbers were reduced by irradiation, but then elevated (past control levels) in monocyte-engrafted mice in both the cortex and hippocampus. These findings highlight not only irradiation-induced deficits in astrocytes, but also compensatory responses for this loss following CSF1Ri-induced monocyte engraftment. Together, these data provide evidence of monocyteastrocyte crosstalk; it could be possible that monocytes are involved in regulating astrocytes or that astrocytes respond to monocyte engraftment in the brain. In line with this, a previous study highlights cross-regulation between astrocyte proliferation and monocyte invasion during scar formation after brain injury. However, contrary to our data, they show that reducing monocyte invasion using $\mathrm{CCR} 2^{-/-}$mice results in elevated reactive astrocyte proliferation [87]. In addition to astrocyte changes, alterations in MAP2 were also found. MAP2 was significantly reduced by irradiation in the cortex and hippocampus and returned to control levels in the hippocampus of monocyte-engrafted mice, similar to previous transcriptional patterns. MAP2 is important for microtubule stabilization, neural plasticity, and normal neuronal cytoskeletal structure; thus, this data could reflect alterations in dendritic spine structure or neurite growth. In agreement, a previous study has shown that cranial irradiation results in significant decreases in dendritic spine density in pyramidal neurons in the hippocampus [88]. Thus, dendritic spine structure or neurite growth may be lost during irradiation and returned to control levels at the facilitation of monocytes in the hippocampus. However, MAP2 also serves as a ciliary marker. In the adult brain, neurons and astrocytes contain one primary cilium, a small microtubule-based signaling process, considered the cellular "antennae" for detecting extracellular signals. Studies have shown that primary cilia are responsible for regulating cell division, development, and tissue regeneration (including DNA damage and repair) $[73,74]$. Further work is needed to explore the effects of irradiation and monocyte engraftment on primary cilia, but these alterations in MAP2 staining could also suggest that replacing microglia with monocytes may help facilitate primary cilia integrity lost due to irradiation. Evaluation of synaptic markers reveals brain region-specific vulnerability to irradiation and monocyte engraftment. In the hippocampus, we observe that irradiation can lead to synaptic deficits, as seen by a loss in $\mathrm{PSD}^{+} 5^{+}$puncta, even $\sim 10$ months after irradiation, which is slightly improved by monocyte engraftment. However, in the cortex, we only observe a significant loss of PSD95 $5^{+}$puncta in monocyte-engrafted mice, indicating that monocytes may phagocytose these synaptic elements. In contrast, a recent report showed that monocytes exhibit a reduced capacity to phagocytose synaptic compartments, although it should be noted that these investigators used a different treatment paradigm (mice were treated for 21 days with CSF1Ri during three rounds of irradiation) and evaluated 4 weeks after irradiation, which could explain the discrepancy. No differences in synaptic marker SV2A were found, indicating that the synaptic alterations observed in monocyte-engrafted mice could be specific to postsynaptic terminals. Since microglia are involved in remodeling synapses, specifically in pruning presynaptic inputs [89], further work is needed to explore and elucidate these long-term engrafted monocyte-synapse interactions.

By behavioral and cognitive task evaluation, we observe few significant differences in behavior in control compared to monocyte-engrafted mice, consistent with previous findings by Cronk et al. that the presence of $48 \%$ CNS engraftment of monocytes does not significantly alter cognitive function [22]. The most prominent changes in behavior were found as a result of irradiation, which lead to alterations in locomotive activity as measured by total distance traveled at 1 month and reduced working memory as measured by a spontaneous alternation task, in both irradiated and monocyte-engrafted mice. In line with these findings, several studies have shown that cranial irradiation disrupts behavioral and cognitive function in rodents, including motor activity and hippocampal and non-hippocampal-dependent cognitive tasks [90, 91]. Interestingly, we do observe a decrease in cortical MAP2 and PSD95 staining, which could indicate brain region-specific vulnerability to irradiation, specifically the cortex. In agreement with this, a recent study has shown that in humans cerebral cortical regions are selectively vulnerable to radiationrelated atrophy [92] and a study in rats showed that radiation induces deficits in cortical synaptic plasticity [93]. Since the spontaneous alternation task relies on various areas of the cortex, as well as the hippocampus, it could be possible that irradiation results in deficits involving the functional integration of these two brain regions. In line with this data, a recent study has shown that following sub-lethal irradiation brain injury, patients experience longitudinal changes in the functional 
connectivity of hippocampal-related cortices [94]. Our study indicates that in the hippocampus, monocyte engraftment can help resolve irradiation-induced deficits, however, in the cortex, may further exacerbate these deficits.

\section{Conclusions}

In this study, we provide evidence that irradiation and monocyte engraftment elicit brain region-dependent alterations and show that irradiation-induced changes can be reversed by the replacement of microglia with monocytes in the hippocampus. Most importantly, we highlight the long-term impact of monocyte engraftment on the CNS. Our data provide evidence that near complete and long-term peripheral myeloid cell engraftment can be achieved, providing a model to deliver therapeutically relevant cells and molecules to the brain; however, the next steps involving therapeutic use should be aware of the differential and sometimes detrimental effects of monocyte engraftment in the CNS. We propose that in developing improved methods of cell delivery to the brain, investigators should focus on methods that do not involve whole-body irradiation. These methods could include promising developments in irradiation technology such as flash irradiation, which are less harmful to the CNS environment. Successful replacement of the microglial tissue with BM-derived cells could have broad implications for many neurological diseases, including those associated with myeloid cell dysfunction, such as Alzheimer's disease and multiple sclerosis, as well as, provide a route for brain-wide therapeutic gene delivery.

\section{Supplementary information}

Supplementary information accompanies this paper at https://doi.org/10. 1186/s12974-020-01931-0.

Additional file 1: Figure S1. Related to Figure 3: Myeloid cell characterization after long-term peripheral myeloid cell engraftment. (A) Representative immunofluorescence and high resolution images of microglial morphology modeling (utilizing the Imaris Filaments module) of myeloid cells in the brains of control (WT CON), irradiated (GFP-BM CON), and monocyte-engrafted (GFP-BM REPOP) mice stained for common myeloid marker ionized calcium binding adaptor molecule 1 (IBA1, blue) and GFP (BM-derived cells, green) in the cortex. (B) Representative immunofluorescence single stain image of TMEM119 (red), a unique marker for microglia. Scale bar: 100 $\mu \mathrm{m}(\mathrm{A}, 20 \mathrm{x})$; $15 \mu \mathrm{m}(\mathrm{A}, 63 \mathrm{x}) ; \sim 75$ (B). Figure S2. Related to Figure 4: Transcriptional changes following long-term peripheral myeloid cell engraftment. (A) Quantification of RPKM values for the top upregulated and downregulated genes in monocyte-engrafted mice across all three brain regions. All RPKM values can be explored at http://rnaseq.mind.uci.edu/green/long-term_monocytes/ (B) Eigengene network displaying the correlation between color modules $(n=32)$. Data are represented as mean $\pm \operatorname{SEM}(n=4)$. Figure S3. Related to Figure 5: The differential and brain region-dependent effects of long-term peripheral myeloid cell engraftment on astrocytic and neuronal properties. (A-B) Representative immunofluorescence single stain images of different astrocyte subtypes stained for $\mathrm{S100 \beta}$ (red, A) and GFAP (red, B) in the hippocampus of control (WT CON), irradiated
(GFP-BM CON), and monocyte-engrafted (GFP-BM REPOP) mice. Scale bar: $\sim 150 \mu \mathrm{m}(\mathrm{A}) ; \sim 75 \mu \mathrm{m}(\mathrm{B}, \mathrm{D}, \mathrm{N}, \mathrm{Q}) ; \sim 60(\mathrm{~S}) ; \sim 25 \mu \mathrm{m}(\mathrm{C})$.

\section{Acknowledgments}

We thank Ayer Darling Jue for the excellent technical assistance and Brian L. West and Andrey Rymar at Plexxikon, Inc., for providing and formulating the CSF1R inhibitor chow.

\section{Authors' contributions}

L.A.H. developed experimental protocols, designed, performed, and analyzed experiments and wrote the manuscript. A.R.N. designed, performed, and analyzed the experiments. Y.G. designed, performed, and analyzed experiments. N.S. performed the bioinformatic analysis. E.H., S.J.K., and A.D.J. performed the experiments. M.A.I. contributed to the project design. K.N.G. directed the project, designed the experiments, interpreted the results, and wrote the manuscript. The author(s) read and approved the final manuscript.

\section{Funding}

This work was supported by the National Institutes of Health $(\mathrm{NHH})$ under awards R01NS083801 (NINDS), R01AG056768 (NIA), and P50AG016573 (NIA) L.A.H. was supported by the Alzheimer's Association Research Fellowship (AARF-16-442762). Y.G. was supported by the NIH T32 training grant (NS082174).

\section{Availability of data and materials}

The Fastq files and processed data matrices were deposited in GEO with the accession ID GSE157593. Gene expression datasets supporting the conclusions of this article are available at http://rnaseq.mind.uci.edu/green/ long-term_monocytes/.

\section{Ethics approval and consent to participate}

Not applicable

\section{Consent for publication}

Not applicable

\section{Competing interests}

The authors declare that they have no competing interests.

\section{Author details}

'Department of Neurobiology and Behavior, University of California, 3208 Biological Sciences III, Irvine, CA 92697-4545, USA. ${ }^{2}$ Sue and Bill Gross Stem Cell Research Center, University of California, Irvine, CA 92697, USA.

${ }^{3}$ Department of Molecular Biology and Biochemistry, University of California, Irvine, CA 92697, USA

Received: 26 May 2020 Accepted: 17 August 2020

Published online: 20 September 2020

\section{References}

1. Nimmerjahn A, Kirchhoff F, Helmchen F. Resting microglial cells are highly dynamic surveillants of brain parenchyma in vivo. Science. 2005;308(5726): $1314-8$

2. Ginhoux F, Greter M, Leboeuf M, Nandi S, See P, Gokhan S, et al. Fate mapping analysis reveals that adult microglia derive from primitive macrophages. Science. 2010;330(6005):841-5.

3. Kierdorf K, Erny D, Goldmann T, Sander V, Schulz C, Perdiguero EG, et al. Microglia emerge from erythromyeloid precursors via Pu.1- and Irf8dependent pathways. Nat Neurosci. 2013;16(3):273-80.

4. Ajami B, Bennett JL, Krieger C, Tetzlaff W, Rossi FM. Local self-renewal can sustain CNS microglia maintenance and function throughout adult life. Nat Neurosci. 2007;10(12):1538-43.

5. Mildner A, Schmidt H, Nitsche M, Merkler D, Hanisch UK, Mack M, et al. Microglia in the adult brain arise from Ly-6ChiCCR2+ monocytes only under defined host conditions. Nat Neurosci. 2007;10(12):1544-53.

6. Ginhoux F, Lim S, Hoeffel G, Low D, Huber T. Origin and differentiation of microglia. Front Cell Neurosci. 2013;7:45.

7. Elmore MR, Najafi AR, Koike MA, Dagher NN, Spangenberg EE, Rice RA, et al. Colony-stimulating factor 1 receptor signaling is necessary for microglia 
viability, unmasking a microglia progenitor cell in the adult brain. Neuron. 2014;82(2):380-97.

8. Spangenberg EE, Lee RJ, Najafi AR, Rice RA, Elmore MR, Blurton-Jones M, et al. Eliminating microglia in Alzheimer's mice prevents neuronal loss without modulating amyloid-beta pathology. Brain. 2016;139(Pt 4):1265-81.

9. Dagher NN, Najafi AR, Kayala KM, Elmore MR, White TE, Medeiros R, et al. Colony-stimulating factor 1 receptor inhibition prevents microglial plaque association and improves cognition in 3xTg-AD mice. J Neuroinflammation. 2015;12:139.

10. Rice RA, Spangenberg EE, Yamate-Morgan H, Lee RJ, Arora RP, Hernandez MX, et al. Elimination of microglia improves functional outcomes following extensive neuronal loss in the hippocampus. J Neurosci. 2015;35(27):997789.

11. Odabas A, McCully CML, Cruz R, Figg WD, Glod J, Rymar A, et al. PHRM-03. Cerebrospinal fluid penetration of pexidartinib (PLX3397), a CSF1R inhibitor, in a nonhuman primate model. Neuro Oncol. 2018;20(Suppl 2):i157-i.

12. Cannarile MA, Weisser M, Jacob W, Jegg AM, Ries CH, Ruttinger D. Colonystimulating factor 1 receptor (CSF1R) inhibitors in cancer therapy. J Immunother Cancer. 2017;5(1):53.

13. Tap WD, Wainberg ZA, Anthony SP, Ibrahim PN, Zhang C, Healey JH, et al. Structure-guided blockade of CSF1R kinase in tenosynovial giant-cell tumor. N Engl J Med. 2015;373(5):428-37.

14. Spangenberg EE, Green KN. Inflammation in Alzheimer's disease: lessons learned from microglia-depletion models. Brain Behav Immun. 2017;61:1-11.

15. Tap WD, Gelderblom H, Palmerini E, Desai J, Bauer S, Blay JY, et al. Pexidartinib versus placebo for advanced tenosynovial giant cell tumour (ENLIVEN): a randomised phase 3 trial. Lancet. 2019;394(10197):478-87.

16. Elmore MR, Lee RJ, West BL, Green KN. Characterizing newly repopulated microglia in the adult mouse: impacts on animal behavior, cell morphology, and neuroinflammation. PLoS One. 2015;10(4):e0122912.

17. Rice RA, Pham J, Lee RJ, Najafi AR, West BL, Green KN. Microglial repopulation resolves inflammation and promotes brain recovery after injury. Glia. 2017;65(6):931-44.

18. Najafi AR, Crapser J, Jiang S, Ng W, Mortazavi A, West BL, et al. A limited capacity for microglial repopulation in the adult brain. Glia. 2018;66(11): 2385-96.

19. Elmore MRP, Hohsfield LA, Kramar EA, Soreq L, Lee RJ, Pham ST, et al. Replacement of microglia in the aged brain reverses cognitive, synaptic, and neuronal deficits in mice. Aging Cell. 2018;17(6):e12832.

20. Zhan L, Sohn PD, Zhou Y, Li Y, Gan L. A Mac2-positive progenitor-like microglial population survives independent of CSF1R signaling in adult mouse brain. bioRxiv. 2019:722090. https://doi.org/10.1101/722090.

21. Huang $Y, X u Z$ Z, Xiong S, Sun F, Qin G, Hu G, et al. Repopulated microglia are solely derived from the proliferation of residual microglia after acute depletion. Nat Neurosci. 2018;21(4):530-40.

22. Cronk JC, Filiano AJ, Louveau A, Marin I, Marsh R, Ji E, et al. Peripherally derived macrophages can engraft the brain independent of irradiation and maintain an identity distinct from microglia. J Exp Med. 2018;215(6):1627-47.

23. Bohlen CJ, Bennett FC, Tucker AF, Collins HY, Mulinyawe SB, Barres BA. Diverse requirements for microglial survival, specification, and function revealed by defined-medium cultures. Neuron. 2017;94(4):759-73.e8.

24. Bennett FC, Bennett ML, Yagoob F, Mulinyawe SB, Grant GA, Hayden Gephart M, et al. A combination of ontogeny and CNS environment establishes microglial identity. Neuron. 2018;98(6):1170-83.e8.

25. Hasselmann J, Coburn MA, England W, Figueroa Velez DX, Kiani Shabestari $\mathrm{S}$, Tu CH, et al. Development of a chimeric model to study and manipulate human microglia in vivo. Neuron. 2019;103(6):1016-1033.e10.

26. Peake K, Manning J, Lewis CA, Barr C, Rossi F, Krieger C. Busulfan as a myelosuppressive agent for generating stable high-level bone marrow chimerism in mice. JoVE. 2015:98:e52553.

27. Prinz $M$, Priller J. Microglia and brain macrophages in the molecular age: from origin to neuropsychiatric disease. Nat Rev Neurosci. 2014;15(5):300-12.

28. Herz J, Filiano AJ, Smith A, Yogev N, Kipnis J. Myeloid cells in the central nervous system. Immunity. 2017:46(6):943-56.

29. Gyoneva S, Kim D, Katsumoto A, Kokiko-Cochran ON, Lamb BT, Ransohoff RM. Ccr2 deletion dissociates cavity size and tau pathology after mild traumatic brain injury. J Neuroinflammation. 2015;12(1):228.

30. Wohleb ES, McKim DB, Sheridan JF, Godbout JP. Monocyte trafficking to the brain with stress and inflammation: a novel axis of immune-tobrain communication that influences mood and behavior. Front Neurosci. 2014;8:447.
31. Yamasaki R, Lu H, Butovsky O, Ohno N, Rietsch AM, Cialic R, et al. Differential roles of microglia and monocytes in the inflamed central nervous system. J Exp Med. 2014;211(8):1533.

32. Mildner A, Mack M, Schmidt H, Bruck W, Djukic M, Zabel MD, et al. CCR2+ Ly-6Chi monocytes are crucial for the effector phase of autoimmunity in the central nervous system. Brain. 2009;132(Pt 9):2487-500.

33. Varvel NH, Neher JJ, Bosch A, Wang W, Ransohoff RM, Miller RJ, et al. Infiltrating monocytes promote brain inflammation and exacerbate neuronal damage after status epilepticus. Proc Natl Acad Sci U S A. 2016; 113(38):E5665-74.

34. Prinz M, Priller J, Sisodia SS, Ransohoff RM. Heterogeneity of CNS myeloid cells and their roles in neurodegeneration. Nat Neurosci. 2011;14(10):122735 .

35. London A, Itskovich E, Benhar I, Kalchenko V, Mack M, Jung S, et al. Neuroprotection and progenitor cell renewal in the injured adult murine retina requires healing monocyte-derived macrophages. J Exp Med. 2011;208(1):23-39.

36. Shechter R, London A, Varol C, Raposo C, Cusimano M, Yovel G, et al. Infiltrating blood-derived macrophages are vital cells playing an anti-inflammatory role in recovery from spinal cord injury in mice. PLoS Med. 2009;6(7):e1000113.

37. Chen SK, Tvrdik P, Peden E, Cho S, Wu S, Spangrude G, et al. Hematopoietic origin of pathological grooming in Hoxb8 mutant mice. Cell. 2010;141(5): $775-85$.

38. Platt FM, Lachmann RH. Treating lysosomal storage disorders: current practice and future prospects. Biochim Biophys Acta. 2009;1793(4):737-45.

39. Derecki NC, Cronk JC, Lu Z, Xu E, Abbott SB, Guyenet PG, et al. Wild-type microglia arrest pathology in a mouse model of Rett syndrome. Nature. 2012;484(7392):105-9.

40. Hsiao EY, McBride SW, Chow J, Mazmanian SK, Patterson PH. Modeling an autism risk factor in mice leads to permanent immune dysregulation. Proc Natl Acad Sci U S A. 2012;109(31):12776-81.

41. Spangenberg E, Severson PL, Hohsfield LA, Crapser J, Zhang J, Burton EA, et al. Sustained microglial depletion with CSF1R inhibitor impairs parenchymal plaque development in an Alzheimer's disease model. Nat Commun. 2019;10(1):3758

42. Dobin A, Davis CA, Schlesinger F, Drenkow J, Zaleski C, Jha S, et al. STAR: ultrafast universal RNA-seq aligner. Bioinformatics. 2013;29(1):15-21.

43. Liao Y, Smyth GK, Shi W. The R package Rsubread is easier, faster, cheaper and better for alignment and quantification of RNA sequencing reads. Nucleic Acids Res. 2019;47(8):e47.

44. Ritchie ME, Phipson B, Wu D, Hu Y, Law CW, Shi W, et al. limma powers differential expression analyses for RNA-sequencing and microarray studies. Nucleic Acids Res. 2015:43(7):e47.

45. Robinson MD, McCarthy DJ, Smyth GK. edgeR: a bioconductor package for differential expression analysis of digital gene expression data. Bioinformatics. 2010;26(1):139-40.

46. McCarthy DJ, Chen Y, Smyth GK. Differential expression analysis of multifactor RNA-Seq experiments with respect to biological variation. Nucleic Acids Res. 2012;40(10):4288-97.

47. Carlson M. org.Mm.eg.db: genome wide annotation for mouse; 2018.

48. Blighe K. EnhancedVolcano: publication-ready volcano plots with enhanced colouring and labeling. R package version 1012019.

49. Zhang B, Horvath S. A general framework for weighted gene co-expression network analysis. Stat Appl Genet Mol Biol. 2005:4:Article17.

50. Lampron A, Lessard M, Rivest S. Effects of myeloablation, peripheral chimerism, and whole-body irradiation on the entry of bone marrowderived cells into the brain. Cell Transplant. 2012;21(6):1149-59.

51. Balentova S, Adamkov M. Molecular, cellular and functional effects of radiationinduced brain injury: a review. Int J Mol Sci. 2015;16(11):27796-815.

52. Lumniczky K, Szatmari T, Safrany G. lonizing radiation-induced immune and inflammatory reactions in the brain. Front Immunol. 2017;8:517.

53. Zhan L, Krabbe G, Du F, Jones I, Reichert MC, Telpoukhovskaia M, et al. Proximal recolonization by self-renewing microglia re-establishes microglial homeostasis in the adult mouse brain. PLoS Biol. 2019;17(2):e3000134.

54. Bruttger J, Karram K, Wortge S, Regen T, Marini F, Hoppmann N, et al. Genetic cell ablation reveals clusters of local self-renewing microglia in the mammalian central nervous system. Immunity. 2015;43(1):92-106.

55. Green KN, Crapser JD, Hohsfield LA. To kill a microglia: a case for CSF1R inhibitors. Trends Immunol. 2020;in press.

56. Waisman A, Ginhoux F, Greter M, Bruttger J. Homeostasis of microglia in the adult brain: review of novel microglia depletion systems. Trends Immunol. 2015;36(10):625-36. 
57. Faraco G, Park L, Anrather J, ladecola C. Brain perivascular macrophages: characterization and functional roles in health and disease. J Mol Med (Berl). 2017;95(11):1143-52.

58. Goldmann T, Wieghofer P, Jordao MJ, Prutek F, Hagemeyer N, Frenzel K, et al. Origin, fate and dynamics of macrophages at central nervous system interfaces. Nat Immunol. 2016;17(7):797-805.

59. Yuan H, Gaber MW, Boyd K, Wilson CM, Kiani MF, Merchant TE. Effects of fractionated radiation on the brain vasculature in a murine model: bloodbrain barrier permeability, astrocyte proliferation, and ultrastructural changes. Int J Radiat Oncol Biol Phys. 2006;66(3):860-6.

60. Zhou K, Bostrom M, Ek CJ, Li T, Xie C, Xu Y, et al. Radiation induces progenitor cell death, microglia activation, and blood-brain barrier damage in the juvenile rat cerebellum. Sci Rep. 2017;7:46181.

61. Qin DX, Zheng R, Tang J, Li JX, Hu YH. Influence of radiation on the bloodbrain barrier and optimum time of chemotherapy. Int J Radiat Oncol Biol Phys. 1990;19(6):1507-10.

62. Park M-K, Kim S, Jung U, Kim I, Kim JK, Roh C. Effect of acute and fractionated irradiation on hippocampal neurogenesis. Molecules. 2012; 17(8):9462-8.

63. Mineyeva OA, Bezriadnov DV, Kedrov AV, Lazutkin AA, Anokhin KV, Enikolopov GN. Radiation induces distinct changes in defined subpopulations of neural stem and progenitor cells in the adult hippocampus. Front Neurosci. 2019;12:1013.

64. Patel AA, Zhang Y, Fullerton JN, Boelen L, Rongvaux A, Maini AA, et al. The fate and lifespan of human monocyte subsets in steady state and systemic inflammation. J Exp Med. 2017;214(7):1913.

65. Reu P, Khosravi A, Bernard S, Mold JE, Salehpour M, Alkass K, et al. The lifespan and turnover of microglia in the human brain. Cell Rep. 2017;20(4): 779-84

66. Dubbelaar ML, Kracht L, Eggen BJL, Boddeke E. The kaleidoscope of microglial phenotypes. Front Immunol. 2018;9:1753.

67. Ayata P, Badimon A, Strasburger HJ, Duff MK, Montgomery SE, Loh Y-HE, et al. Epigenetic regulation of brain region-specific microglia clearance activity. Nat Neurosci. 2018;21(8):1049-60.

68. Tan Y-L, Yuan Y, Tian L. Microglial regional heterogeneity and its role in the brain. Mol Psychiatry. 2020;25(2):351-67.

69. De Biase LM, Schuebel KE, Fusfeld ZH, Jair K, Hawes IA, Cimbro R, et al. Local cues establish and maintain region-specific phenotypes of basal ganglia microglia. Neuron. 2017;95(2):341-56.e6.

70. Grabert K, Michoel T, Karavolos MH, Clohisey S, Baillie JK, Stevens MP, et al. Microglial brain region-dependent diversity and selective regional sensitivities to aging. Nat Neurosci. 2016;19(3):504-16.

71. Willers H, Dahm-Daphi J, Powell SN. Repair of radiation damage to DNA. Br J Cancer. 2004;90(7):1297-301.

72. Shkreta L, Chabot B. The RNA splicing response to DNA damage. Biomolecules. 2015:5(4):2935-77.

73. Sterpka A, Chen X. Neuronal and astrocytic primary cilia in the mature brain Pharmacol Res. 2018:137:114-21.

74. Yuan S, Sun Z. Expanding horizons: ciliary proteins reach beyond cilia. Annu Rev Genet. 2013:47:353-76.

75. Raber J, Torres ERS, Akinyeke T, Lee J, Weber Boutros SJ, Turker MS, et al. Detrimental effects of helium ion irradiation on cognitive performance and cortical levels of MAP-2 in B6D2F1 mice. Int J Mol Sci. 2018;19(4):1247.

76. Johnson GV, Jope RS. The role of microtubule-associated protein 2 (MAP-2) in neuronal growth, plasticity, and degeneration. J Neurosci Res. 1992;33(4): 505-12.

77. Kang J, Kim W, Seo H, Kim E, Son B, Lee S, et al. Radiation-induced overexpression of transthyretin inhibits retinol-mediated hippocampal neurogenesis. Sci Rep. 2018:8(1):8394.

78. Bajjalieh SM, Frantz GD, Weimann JM, McConnell SK, Scheller RH. Differential expression of synaptic vesicle protein 2 (SV2) isoforms. J Neurosci. 1994; 14(9):5223-35

79. Miedel CJ, Patton JM, Miedel AN, Miedel ES, Levenson JM. Assessment of spontaneous alternation, novel object recognition and limb clasping in transgenic mouse models of amyloid- $\beta$ and tau neuropathology. J Vis Exp. 2017:123:55523.

80. Han J, Harris RA, Zhang XM. An updated assessment of microglia depletion: current concepts and future directions. Mol Brain. 2017;10(1):25.

81. Menzel F, Kaiser N, Haehnel S, Rapp F, Patties I, Schoneberg N, et al. Impact of X-irradiation on microglia. Glia. 2018;66(1):15-33.
82. Feng X, Chen D, Gupta S, Liu S, Gupta N, Rosi S. Replacement of microglia by monocyte-derived macrophages prevents long-term memory deficits after therapeutic irradiation. bioRxiv. 2019:794354.

83. Varvel NH, Grathwohl SA, Baumann F, Liebig C, Bosch A, Brawek B, et al. Microglial repopulation model reveals a robust homeostatic process for replacing CNS myeloid cells. Proc Natl Acad Sci U S A. 2012;109(44):18150-5.

84. Lund H, Pieber M, Parsa R, Han J, Grommisch D, Ewing E, et al. Competitive repopulation of an empty microglial niche yields functionally distinct subsets of microglia-like cells. Nat Commun. 2018;9(1):4845.

85. Shemer A, Grozovski J, Tay TL, Tao J, Volaski A, Süß P, et al. Engrafted parenchymal brain macrophages differ from microglia in transcriptome, chromatin landscape and response to challenge. Nat Commun. 2018;9(1): 5206.

86. Dietrich J, Baryawno N, Nayyar N, Valtis YK, Yang B, Ly I, et al. Bone marrow drives central nervous system regeneration after radiation injury. J Clin Invest. 2018;128(1):281-93.

87. Frik J, Merl-Pham J, Plesnila N, Mattugini N, Kjell J, Kraska J, et al. Cross-talk between monocyte invasion and astrocyte proliferation regulates scarring in brain injury. EMBO Rep. 2018;19(5):e45294.

88. Chakraborti A, Allen A, Allen B, Rosi S, Fike JR. Cranial irradiation alters dendritic spine density and morphology in the hippocampus. PLoS One. 2012;7(7):e40844.

89. Schafer DP, Lehrman EK, Kautzman AG, Koyama R, Mardinly AR, Yamasaki R, et al. Microglia sculpt postnatal neural circuits in an activity and complement-dependent manner. Neuron. 2012;74(4):691-705.

90. Son Y, Yang M, Wang H, Moon C. Hippocampal dysfunctions caused by cranial irradiation: a review of the experimental evidence. Brain Behav Immun. 2015;45:287-96

91. Tome WA, Gokhan S, Gulinello ME, Brodin NP, Heard J, Mehler MF, et al. Hippocampal-dependent neurocognitive impairment following cranial irradiation observed in pre-clinical models: current knowledge and possible future directions. Br J Radiol. 2016;89(1057):20150762.

92. Seibert TM, Karunamuni R, Kaifi S, Burkeen J, Connor M, Krishnan AP, et al. Cerebral cortex regions selectively vulnerable to radiation dose-dependent atrophy. Int J Radiat Oncol Biol Phys. 2017;97(5):910-8.

93. Zhang D, Zhou W, Lam TT, Weng C, Bronk L, Ma D, et al. Radiation induces age-dependent deficits in cortical synaptic plasticity. Neuro Oncol. 2018; 20(9):1207-14

94. Chen SC-J, Abe Y, Fang P-T, Hsieh Y-J, Yang Y-I, Lu T-Y, et al. Prognosis of hippocampal function after sub-lethal irradiation brain injury in patients with nasopharyngeal carcinoma. Sci Rep. 2017;7(1):14697.

\section{Publisher's Note}

Springer Nature remains neutral with regard to jurisdictional claims in published maps and institutional affiliations.

Ready to submit your research? Choose BMC and benefit from:

- fast, convenient online submission

- thorough peer review by experienced researchers in your field

- rapid publication on acceptance

- support for research data, including large and complex data types

- gold Open Access which fosters wider collaboration and increased citations

- maximum visibility for your research: over $100 \mathrm{M}$ website views per year

At BMC, research is always in progress.

Learn more biomedcentral.com/submission 